\title{
The Use of Sonophoresis in the Administration of Drugs Throughout the Skin
}

\author{
José Juan Escobar-Chávez ${ }^{1}$, Dalia Bonilla-Martínez², Martha Angélica Villegas-González², Isabel \\ Marlen Rodríguez-Cruz ${ }^{1}$, Clara Luisa Domínguez-Delgado'.
}

${ }^{1}$ División de Estudios de Posgrado (Tecnología Farmacéutica), Facultad de Estudios Superiores CuautitlánUniversidad Nacional Autónoma de México, Cuautitlán Izcalli, Estado de México, México 54740. ${ }^{2}$ División de Ciencias Químicas, Sección de Química Analítica, Facultad de Estudios Superiores Cuautitlán-Universidad Nacional Autónoma de México, Cuautitlán Izcalli, Estado de México, México 54740

Received January 23, 2009; Revised, April 2, 2009; Accepted, April 14, 2009; Published April 16, 2009.

\begin{abstract}
Transdermal drug delivery offers an attractive alternative to the conventional drug delivery methods of oral administration and injection. However, the stratum corneum acts as a barrier that limits the penetration of substances through the skin. Application of ultrasound to the skin increases its permeability (sonophoresis) and enables the delivery of various substances into and through the skin. Ultrasound has been used extensively for medical diagnostics and to a certain extent in medical therapy (physiotherapy, ultrasonic surgery, hyperthermia). Nevertheless, it has only recently become popular as a technique to enhance drug release from drug delivery systems. A number of studies suggest the use of ultrasound as an external mean of delivering drugs at increased rates and at desired times. This review presents the main findings in the field of sonophoresis, namely transdermal drug delivery and transdermal monitoring. Particular attention is paid to proposed enhancement mechanisms and trends in the field of topical and transdermal delivery.
\end{abstract}

\section{INTRODUCTION}

Therapeutic applications of ultrasound pre-date its use as an imaging technique. It was recognized in 1927 that ultrasound (ULTS) could produce lasting changes in biological systems, and this was the start of both safety studies, and of ultrasound therapy [1].

Absorption of ultrasonic energy leads to tissue heating, and this has been used with therapeutic intent in many conditions. More recently it has been realized that benefit may also be obtained from the non-thermal effects that occur as ultrasound travels through tissue.

ULTS therapies can broadly be divided into "high" power and "low" power therapies where high power applications include high intensity focused ULTS and lithotripsy, and low power encompasses sonophoresis, sonoporation, gene therapy and bone healing. Apart from physiotherapy uses, ULTS therapies are currently not widespread.

ULTS has been used in the medical field for several decades and can be divided into three major categories: i) high frequency $(2-10 \mathrm{MHz})$ or diagnostic ultrasound,

ii) medium frequency $(0.7-2 \mathrm{MHz})$ or therapeutic ULTS, and

iii) low frequency (5 to $100 \mathrm{KHz}$ ) or power ULTS.[2]

The principal medical applications of ULTS include its use:

a. as a diagnostic tool at various anatomical sites (eyes and orbit, head, neck, chest, breast, abdomen, and pelvis),

b. for physical therapy,

c. in surgery, and

d. in dentistry.

Corresponding Author: José Juan Escobar-Chávez, División de Estudios de Posgrado (Tecnología Farmacéutica), Facultad de Estudios Superiores Cuautitlán-Universidad Nacional Autónoma de México, Cuautitlán Izcalli, Estado de México, México. Email: josejuanescobar@gmail.com 
ULTS was initially investigated for treating localized skin conditions [3] and joint inflammation.[4] More recently, there has been considerable interest in developing ULTS as a technique to enhance transdermal drug delivery [5] and to enable the relatively noninvasive extraction of analytes from the blood [6] The scope of this article is, first, to detail the relationship between ultrasound frequency and improved molecular transport across the skin; second, to describe the mechanisms by which ULTS enhances transdermal transport; and third, to review the increasing sonophoretic enhancement literature to assess the potential for practical transdermal applications in drug delivery and noninvasive clinical chemistry.

A number of excellent reviews that have been published contain detailed discussions concerning many aspects of sonophoresis [7-12]. The present review shows an updated overview of the use of sonophoresis in the pharmaceutical field, specifically in the area of topical and transdermal drugs. This focus is justified due to the magnitude of the experimental data available with the use of this technique. The use of sonophoresis in experimental medicine and pharmaceutical sciences has a long history.

\section{The skin}

The skin is the largest organ of the body [13-15], accounting for more than $10 \%$ of body mass, and the one that enables the body to interact more intimately with its environment. Essentially, the skin consists of four layers: The SC, that is the outer layer of the skin (non-viable epidermis), and forms the rate-controlling barrier for diffusion for almost all compounds. It is composed of dead flattened, keratin-rich cells, the corneocytes. These dense cells are surrounded by a complex mixture of intercellular lipids, namely, ceramides, free fatty acids, cholesterol, and cholesterol sulphate. Their most important feature is that they are structured as ordered bilayer arrays [16]. The predominant diffusional path for a molecule crossing the SC appears to be intercellular [17-19]. The other layers are: the remaining layers of the epidermis (viable epidermis), the dermis, and the subcutaneous tissues (Figure 1). There are also several associated appendages: hair follicles sweat ducts, apocrine glands and nails. In a general context, the skin's functions may be classified as protective, homeostasis maintaining functions, or sensing [20,21].

Many agents are applied to the skin either deliberately or accidentally, with either beneficial or deleterious outcomes. The main interest in dermal absorption assessment is related to: a) Local effects in dermatology (e.g., corticosteroids for dermatitis); b) transport through the skin seeking a systemic effect (e.g., nicotine patches, hormonal drug patches, etc.); c) surface effects (e.g., sunscreens, cosmetics, and anti-infectives) [22]; d) targeting of deeper tissues (e.g., nonsteroidal anti-inflammatory agents) [23-32]; and e) unwanted absorption (e.g., solvents in the workplace, pesticides or allergens) $[33,34]$. Figure 2 summarizes the process of percutaneous absorption.

\section{The Ultrasound}

Sound is a form of mechanical energy that is propagated from one point to another by the interaction between neighboring oscillating particles [35]. The direction of propagation is parallel to the direction of oscillation and, hence, sound is defined as a longitudinal wave. Because its propagation depends entirely on the creation of alternating regions of molecular compression and rarefaction, sound cannot exist in a vacuum. The pressure variation has the same propagation speed and frequency as the oscillations of the molecules about their equilibrium positions. Acoustic waves with frequencies between $20 \mathrm{~Hz}$ and $\sim 20 \mathrm{KHz}$ fall in the audible range. The term ultrasonic refers to sound waves whose frequency is $>20 \mathrm{KHz}$. The intensity (I, expressed in $\mathrm{W} / \mathrm{cm}^{2}$ ), or concentration of power within a specific area in an ULTS beam, is proportional to the square of the amplitude, p, which is the maximum increase or decrease in the pressure relative to ambient conditions in the absence of the sound wave. The complete relationship is:

$$
\mathrm{I}=\mathrm{p}^{2} / 2 \rho \mathrm{c}
$$

where $\rho$ is the density of the medium and $\mathrm{c}$ is the speed of the sound (in human soft tissue, this velocity is $1540 \mathrm{~m} / \mathrm{s}$ ). 


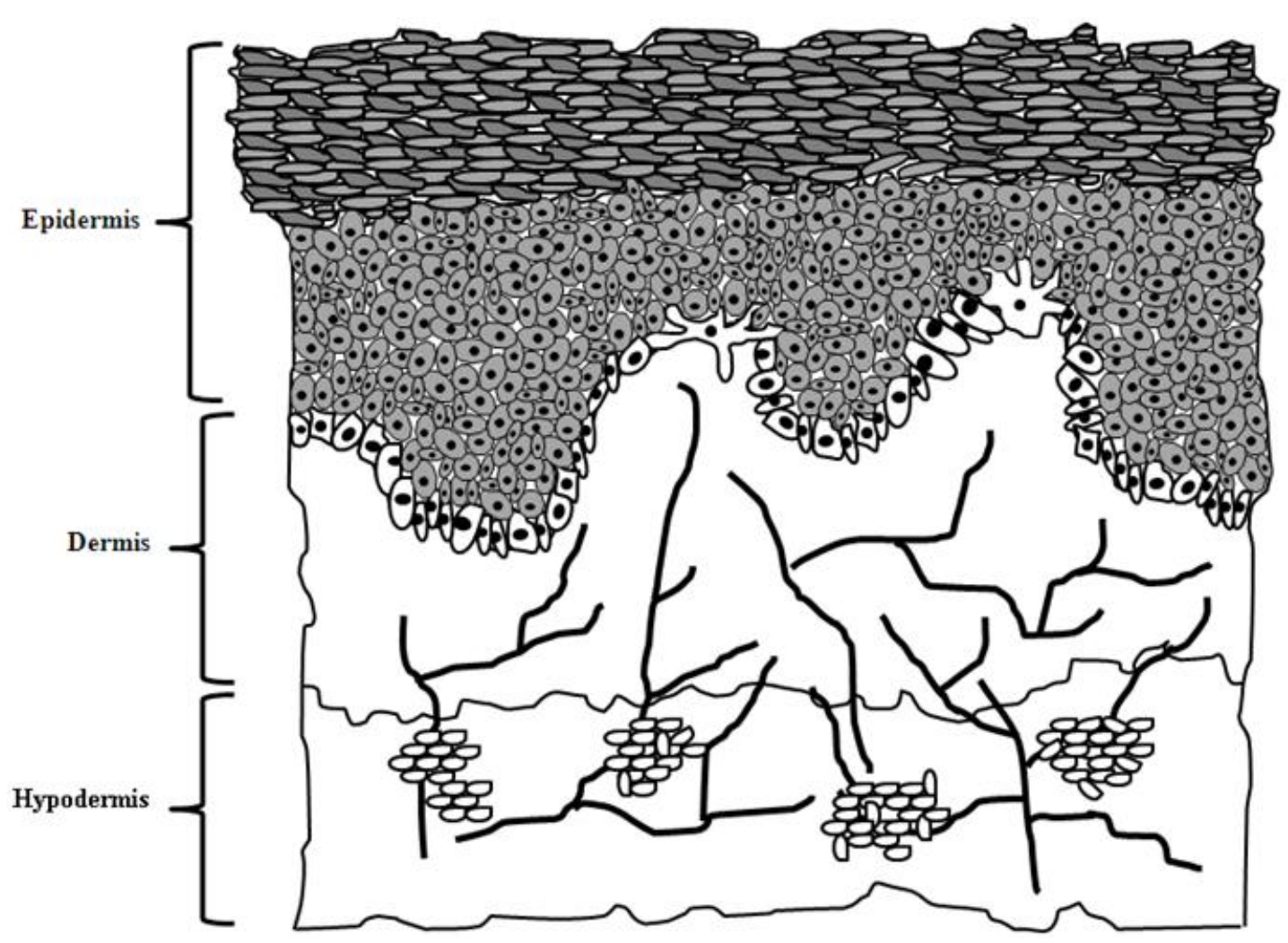

Figure 1. Schematic representation of the skin layers

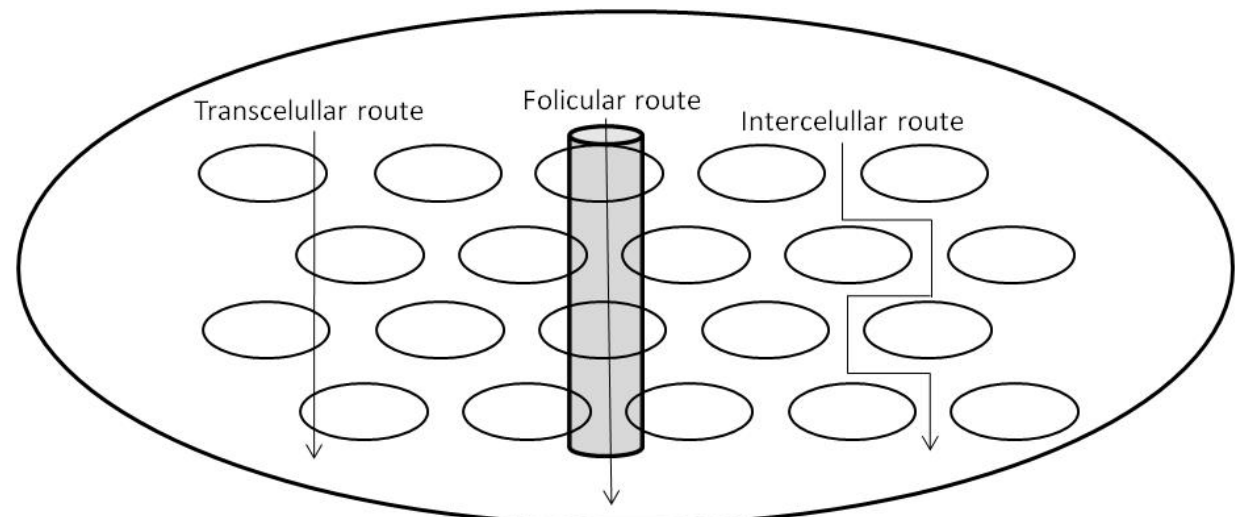

Figure 2. Processes of percutaneous absorption and transdermal delivery

The skin became popular as a potential site for systemic drug delivery, on the one hand, because of the possibility of avoiding the problems of stomach emptying, $\mathrm{pH}$ effects, enzyme deactivation associated with gastrointestinal passage, and hepatic first-pass metabolism; and on the other hand, due to its capability to enable input control. The intensity is progressively lost when a sound wave passes through the body or is deviated from its initial direction, a phenomenon referred to as attenuation. In homogeneous tissue, the attenuation occurs as a result of absorption, in which case the sound energy is transformed into heat and scattered. The source of sound waves in a biomedical ULTS device is a 
piezoelectric crystal transducer. The crystal material may be quartz or another polycrystalline material, such as lead-zirconate-titanium or barium titanate [36]. The sound waves are produced in response to an electrical impulse in the piezoelectric crystal, allowing the conversion of electrical into mechanical or vibrational energy; this transformation requires a molecular medium (solid, liquid, or gas) to be effective. Following the external perturbation, groups of molecules oscillate in phase and transmit their kinetic energy to nearby molecules [2]. The ULTS beam is composed of two fields, the "near field," in the region closest to the transducer face, and the "far field," corresponding to the conical diverging portion of the beam (see Figure 3). The parameters controlling this configuration of the ULTS beam are principally the frequency and the size of transducer [36].

\section{Mechanisms of Action}

Although considerable attention has been given to the investigation of sonophoresis in the past years, its mechanisms were not clearly understood, reflecting the fact that several phenomena may occur in the skin upon ULTS exposure. These include:
a) Cavitation effects.
b) Thermal effects.
c) Induction of convective transport.
d) Mechanical effects.

\section{a) Cavitation effects}

Cavitation is the formation of gaseous cavities in a medium ULTS exposure (Figure 4). The primary cause of cavitation is ULTS-induced pressure variation in the medium. Cavitation involves either the rapid growth and collapse of a bubble (inertial cavitation), or the slow oscillatory motion of a bubble in an ULTS field (stable cavitation). Collapse of cavitation bubbles releases a shock wave that can cause structural alteration in the surrounding tissue [37]. ULTS can generate violent microstreams, which increase the bioavailability of the drugs [38]. Tissues contain air pockets that are trapped in the fibrous structures that act as nuclei for cavitation upon ultrasound exposure. The cavitational effects vary inversely with ULTS frequency and directly with ULTS intensity. Cavitation might be important when low-frequency ULTS is used, gassy fluids are exposed or when small gas-filled spaces are exposed.

Cavitation occurs due to the nucleation of small gaseous cavities during the negative pressure cycles of ULTS, followed by the growth of these bubbles throughout subsequent pressure cycles. Whenever small gaseous nuclei already exist in a medium, cavitation takes place preferentially at those nuclei [39-41]. This cavitation leads to the disordering of the lipid bilayers and formation of aqueous channels in the skin through which drugs can permeate [42-44].

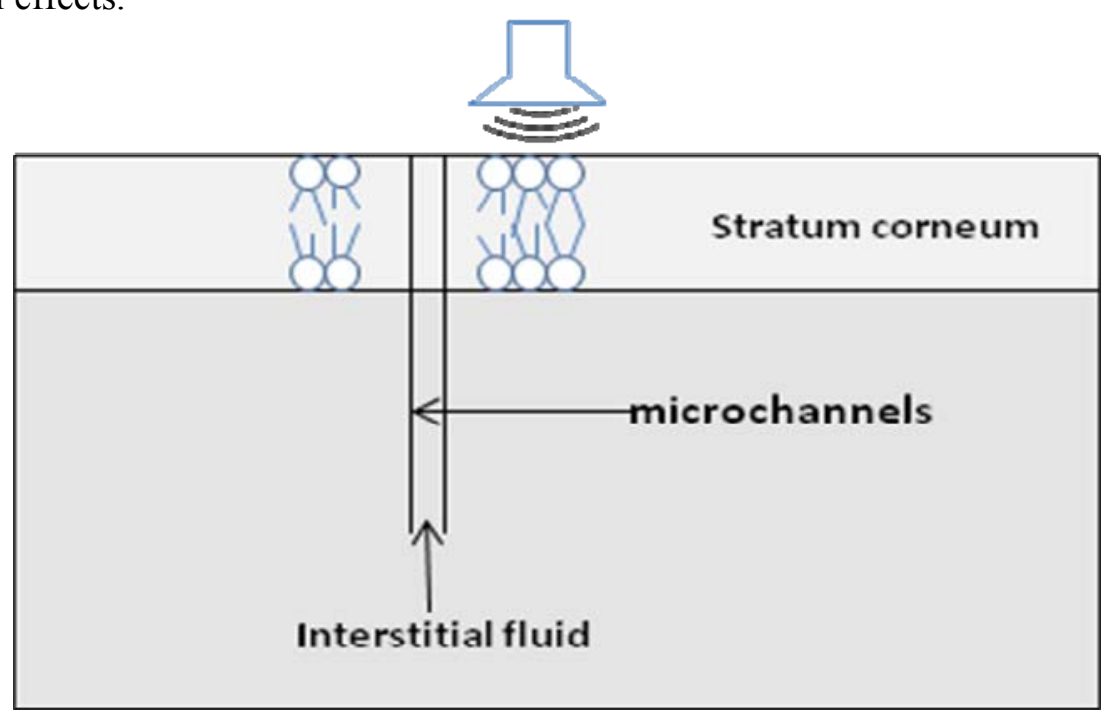

Figure 3. Picture of how ultrasound disrupts the stratum corneum 


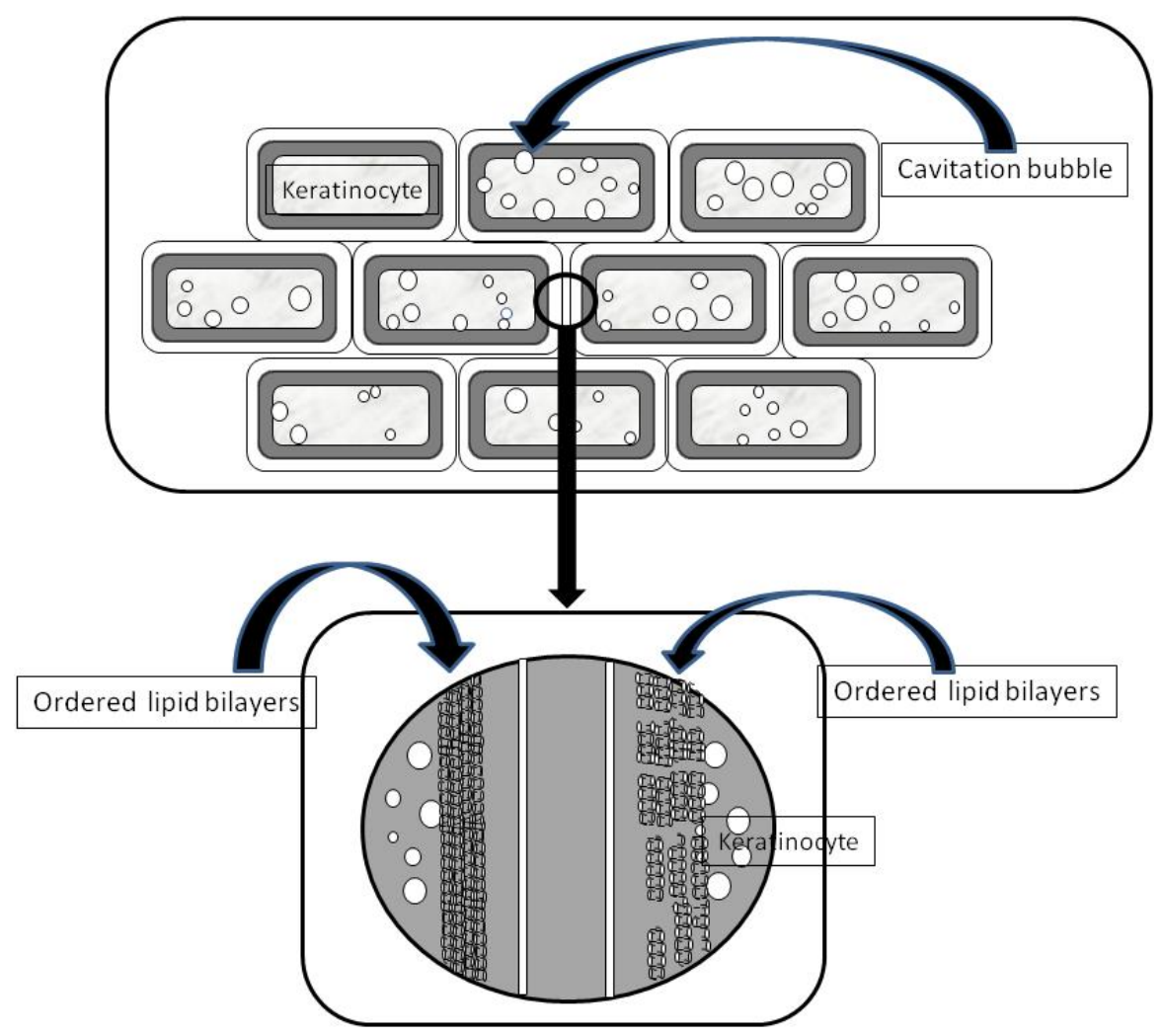

Figure 4. Schematic sketch of cavitation occurring in the keratinocytes. Cavitation occurs preferentially at the interface between the keratinocytes and the lipid bilayers

\section{b) Thermal effects}

Absorption of ULTS increases temperature of the medium. Materials that possess higher ULTS absorption coefficients, such as bone, experience severe thermal effects compared with muscle tissue, which has a lower absorption coefficient [45]. The increase in the temperature of the medium upon ULTS exposure at a given frequency varies directly with the ULTS intensity and exposure time. The absorption coefficient of a medium increases directly with ULTS frequency resulting in temperature increase.

A study [46] suggested the use of a new safety parameter, time to threshold (TT). TT indicates the time after which a threshold temperature rise is exceeded, and how long a piece of tissue can be safely exposed to ULTS, provided the safe threshold is known.

\section{c) Convective transport}

Fluid velocities are generated in porous medium exposed to ULTS due to interference of the incident and reflected ULTS waves in the diffusion cell and oscillations of the cavitation bubbles. Fluid velocities generated in this way may affect transdermal transport by inducing convective transport of the permeant across the skin, especially through hair follicles and sweat ducts. Experimental findings suggest that convective transport does not play an important role in the observed transdermal enhancement [39].

\section{d) Mechanical effects}

ULTS is a longitudinal pressure wave inducing sinusoidal pressure variations in the skin, which, in turn, induce sinusoidal density variation. At frequencies greater than $1 \mathrm{MHz}$, the density variations occur so rapidly that a small gaseous nucleus can not grow and cavitational effects cease. 
But other effects due to density variations, such as generation of cyclic stresses because of density changes that ultimately lead to fatigue of the medium, may continue to occur. Lipid bilayers, being self-assembled structures, can easily be disordered by these stresses, which result in an increase in the bilayer permeability. This increase is, however, non-significant and hence mechanical effects do not play an important role in therapeutic sonophoresis. Thus cavitation induced lipid bilayer disordering is found to be the most important cause for ultrasonic enhancement of transdermal transport [39].

\section{Advantages and disadvantages of sonophoresis}

Sonophoresis is capable of expanding the range of compounds that can be delivered transdermally [4749] (See Figure 5). In addition to the benefits of avoiding the hepatic first-pass effect, and higher patient compliance, the additional advantages [50] and disadvantages that the sonophoretic technique offers can be summarized as follows in Table 1.

\section{Applications of ultrasound}

The unique and promising release of drugs by sonophoresis renders it an attractive candidate as a physical enhancer to administer drugs throughout the skin [52-84]. This is emphasized in Table 2, which summarizes the research on sonophoresis uses in the transdermal administration of drugs.

\section{Analgesic and anti-inflammatory drugs}

Meshali et al. [52] evaluated the effect of intensity, mode, and duration of ULTS application on the transport of three nonsteroidal anti-inflammatory drugs (NSAIDs) across cellulose membrane and rabbit-skin. Ibuprofen, piroxicam and diclofenac sodium were used as the model drugs. Studies were performed in vitro using a modified Franz diffusion assembly adapted to a therapeutic ULTS transducer. ULTS had a significant and positive effect on the transport of the model NSAIDs across cellulose and rabbit skin membranes. Increasing ULTS intensity from 0.5 to $3.0 \mathrm{~W} / \mathrm{cm}^{2}$ led to a proportional increase in drug transport. Continuous ULTS mode was more effective in enhancing drug transport than the pulsed mode. Diclofenac sodium had the least flux and permeability coefficient. This was attributed to its comparatively lower $\mathrm{pKa}$ value that renders the drug more ionizable in the buffer solution, consequently reducing its selective penetration through the membranes. This study demonstrated the therapeutic potential of ULTS in transdermal delivery of NSAIDs and the synergistic effect of temperature and ultrasound operational parameters on drug transport.

(A) Macro Level

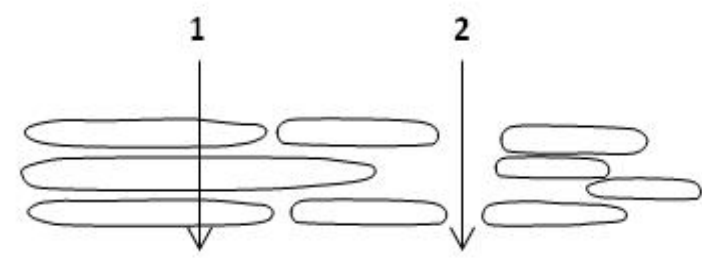

(B) Micro Level

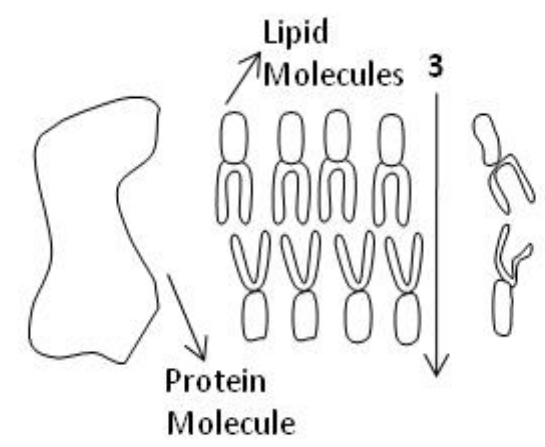

Figure 5. At the macro level (A), phonophoresis is thought to enhance drug penetration through the stratum corneum transcelullary (1), in a process termed acoustic streaming, or through increased pore size (2), in a process termed ultrasonic stirring. At the microlevel (3) disruption of the lipids.

The effect of an ULTS (1 MHz) on transdermal absorption of indomethacin from an ointment was studied in rats by Miyazaki et al. [53]. ULTS energy was supplied for between 5 and $20 \mathrm{~min}$ at a range of intensities $\left(0.25,0.5,0.75\right.$, and $\left.1 \mathrm{~W} \mathrm{~cm}^{-2}\right)$, energy levels commonly used for therapeutic purposes. For evaluating skin penetration of indomethacin, the change of plasma concentration was measured. The pronounced effect of ultrasound on the transdermal absorption of indomethacin was observed at all ultrasound energy levels studied. 
Table 1. Advantages and disadvantages of using sonophoresis as a physical penetration enhancer

Advantages

Enhanced drug penetration (of selected drugs) over passive transport

Allows strict control of transdermal penetration rates

Permits rapid termination of drug delivery through termination of ULTS

Skin remains intact, therefore low risk of introducing infection

Less anxiety provoking or painful than injection

In many cases, greater patient satisfaction

Not immunologically sensitizing

Less risk of systemic absorption than injection

The intensity and the time of application were found to play an important role in the transdermal phonophoretic delivery system of indomethacin; $0.75 \mathrm{~W} \mathrm{~cm}^{-2}$ appeared to be the most effective intensity in improving the transdermal absorption of indomethacin, while the $10 \mathrm{~min}$ ULTS treatment was the most effective. Although the highest penetration was observed at an intensity of $0.75 \mathrm{~W} \mathrm{~cm}^{-2}, 0.5 \mathrm{~W} \mathrm{~cm}$ was preferred because intensities of less than $0.5 \mathrm{~W} \mathrm{~cm}^{-2}$ of ULTS for 10 min did not result in any significant skin temperature rise nor did it have any destructive effect on rat skin. Progressively more skin damage was noted as the intensity and the time of application of ULTS increased.

Tiwari et al. [54] studied the influence of ULTS on percutaneous absorption of ketorolac tromethamine in vitro across rat skin. Sonication was carried out with a continuous mode, at an intensity of $1-3 \mathrm{~W} / \mathrm{cm}^{2}$ and a frequency of $1 \mathrm{MHz}$ for $30 \mathrm{~min}$. A significant increase in permeation of ketorolac through rat skin was observed with the

\section{Disadvantages}

Can be time-consuming to administer

Minor tingling, irritation, and burning have been reported (these effects can often be minimized or eradicated with proper ULTS adjustment [51])

$\mathrm{SC}$ must be intact for effective drug penetration applied sonication at $3 \mathrm{~W} / \mathrm{cm}^{2}$ when compared with permeation at 1 and $2 \mathrm{~W} / \mathrm{cm}^{2}$. Enhanced ketorolac penetration at $3 \mathrm{~W} / \mathrm{cm}^{2}$ can be explained by the mechanical and/or thermal action of ULTS waves. The distance of the ULTS probe from the skin surface did not influence the flux of the drug. Pretreatment of skin by $5 \%$ d-limonene in ethanol for $2 \mathrm{hr}$ followed by sonication at $3 \mathrm{~W} / \mathrm{cm}^{2}(30 \mathrm{~min})$ significantly enhanced the permeation of ketorolac when compared with passive flux with or without enhancer pretreatment.

The aim of Yang et al. [55] was to determine if a ketorolac tromethamine (KT) gel solution could be administered in vivo via phonophoretic transdermal delivery using pulsed ULTS by examining its anti-hyperalgesic and antiinflammatory effects in a rat carrageenan inflammation model. $1 \%$ carrageenan was injected into the plantar surface of the right hind paw of a rat, and anti-hyperalgesic and anti-inflammatory effects of KT via phonophoretic transdermal delivery were examined. 
Table. 2 Research on uses of sonophoresis to administer different drugs through the skin

\section{Analgesic and anti-inflammatory drugs}

\section{Research}

Effect of intensity, mode, and duration of ultrasound application on the transport of three no steroidal antiinflammatory drugs (NSAIDs) across cellulose membrane and rabbit-skin.

The effect of an ultrasound ( $1 \mathrm{MHz}$ ) on transdermal absorption of indomethacin from an ointment in rats.

Study of the influence of ultrasound on percutaneous absorption of ketorolac tromethamine in vitro across rat skin.

To determine if a ketorolac tromethamine (KT) gel solution could be administered in vivo via phonophoretic transdermal delivery using pulsed ultrasound by examining its anti-hyperalgesic and antiinflammatory effects in a rat carrageenan inflammation model.

Application of ultraphonophoresis of $5 \%$ ibuprofen nurofen gel to affected joints of 20 patients.

Examination of the therapeutic effects of phonophoresis with ketoprofen in gel form in patients with enthesopathy of the elbow.

Quantitative study of sodium diclofenac (Voltaren Emulgel, Novartis) phonophoresis in humans.

\section{Outcome}

This study demonstrated the therapeutic potential of ultrasound in transdermal delivery of NSAIDs and the synergistic effect of temperature and ultrasound operational parameters on drug transport.

The intensity and the time of application were found to play an important role in the transdermal phonophoretic delivery system of indomethacin; $0.75 \mathrm{~W} \mathrm{~cm}^{-2}$ appeared to be the most effective intensity in improving the transdermal absorption of indomethacin, while the $10 \mathrm{~min}$ ultrasound treatment was the most effective.

A significant increase in permeation of ketorolac through rat skin was observed with the applied sonication at $3 \mathrm{~W} / \mathrm{cm}^{2}$ when compared with permeation at 1 and 2 $\mathrm{W} / \mathrm{cm}^{2}$.

The transdermal application of KT gel using phonophoresis had significant antihyperalgesic and anti-inflammatory effects. These findings suggest that the transdermal administration of a KT gel using phonophoresis with pulsed ULTS might be useful for treating acute inflammation and pain.

Analgesic efficacy of transcutaneous 5\% gel nurofen in osteoarthrosis and a rise of this efficacy in combined use of ULTS and this medicine in ultraphonophoresis.

The pain symptoms in the elbow resolved in most of the patients. There were statistically significant differences between phonophoresis and ULTS therapy.

The authors conclude that previously applied therapeutic ultrasound irradiation enhances the percutaneous penetration of the topical diclofenac gel, although the mechanism remains unclear.
Miyazaki et al [53]

Tiwari et al. [54]

Yang et al. [55]

Serikov et al. [56]

Cabak et al. [57]

Rosim et al. [58] 


\section{Table 2 Continued.....}

To investigate the in vitro penetration and the in vivo transport of flufenamic acid in dependence of ULTS.
Using this in vitro model they noted it is possible to compare the transdermal penetration and absorption of commercial flufenamic ointment in volunteers.
Hippius et al. [59]

\section{Anesthetics}

Permeation of procaine hydrochloride through cell monolayers applying therapeutical ultrasound.

Extent and velocity of the permeation of procaine hydrochloride through MDCK monolayer can be controlled by phonophoresis.

\section{Antibiotics}

Investigated the topical transport of Cyclosporin A using low-frequency ULTS throughout rat skin.

Evaluation of the efficacy of low frequency sonophoresis (LFS) at $25 \mathrm{KHz}$ produced by a sonicator apparatus for treatment of alopecia areata, melasma and solar lentigo.

Total of 451 experiments (130 with the method of electrophoresis and 321 with the method of phonophoresis) on healthy rabbits of the same species, age, weight and sex with the use of the galvanization apparatus "Potok-1" and ULTS therapeutic apparatus "VTP-1" to administer tetracycline.
The enhanced skin accumulation of Cyclosporin A by the combination of lowfrequency ULTS and chemical enhancers could help significantly to optimize the targeting of the drug without of a concomitant increase of the systemic side effects.

The study showed that LFS, a not aggressive technique, enhanced penetration of topic agents obtaining effects at the level of the epidermis, dermis and appendages (intradermal delivery), giving better results in the treatment of some cosmetic skin disorders.

It was found that the tissue levels of tetracycline administered with the modified methods of electro- and phonophoresis increased with an increase in the current density or ultrasound intensity, the procedure time and antibiotic concentration.

\section{Liu et al.[63]}

Hehn et al. [62]
Ultrasound exposure to the legs of hairless

mice along with topical 2\% lidocaine

solution rapidly induced an anesthetic

effect.

$\begin{array}{ll}\begin{array}{l}\text { Administration of lidocaine } \\ \text { hydrochloride trandermally on healthy } \\ \text { volunteers applying } 0.5 \mathrm{MHz} \text { ULTS. }\end{array} & \begin{array}{l}0.5 \mathrm{MHz} \text { ultrasound in phonophoresis for } \\ \text { conduction anesthesia using lidocaine } \\ \text { hydrochloride for a nerve block, it is more } \\ \text { effective than the } 1 \mathrm{Mhz} \text { that is widely } \\ \text { used in clinical situations. }\end{array}\end{array}$

$\begin{array}{ll}\begin{array}{l}\text { Administration of lidocaine } \\ \text { hydrochloride trandermally on healthy }\end{array} & \begin{array}{l}0.5 \mathrm{MHz} \text { ultrasound in phonophoresis } \\ \text { conduction anesthesia using lidocaine } \\ \text { volunteers applying } 0.5 \mathrm{MHz} \text { ULTS. }\end{array} \\ \begin{array}{l}\text { hydrochloride for a nerve block, it is mor } \\ \text { effective than the } 1 \mathrm{Mhz} \text { that is widely } \\ \text { used in clinical situations. }\end{array}\end{array}$

$\begin{array}{ll}\begin{array}{l}\text { Administration of lidocaine } \\ \text { hydrochloride trandermally on healthy } \\ \text { volunteers applying } 0.5 \mathrm{MHz} \text { ULTS. }\end{array} & \begin{array}{l}0.5 \mathrm{MHz} \text { ultrasound in phonophoresis for } \\ \text { conduction anesthesia using lidocaine } \\ \text { hydrochloride for a nerve block, it is more } \\ \text { effective than the } 1 \mathrm{Mhz} \text { that is widely } \\ \text { used in clinical situations. }\end{array}\end{array}$

$\begin{array}{ll}\begin{array}{l}\text { Administration of lidocaine } \\ \text { hydrochloride trandermally on healthy }\end{array} & \begin{array}{l}0.5 \mathrm{MHz} \text { ultrasound in phonophoresis } \\ \text { conduction anesthesia using lidocaine } \\ \text { volunteers applying } 0.5 \mathrm{MHz} \text { ULTS. }\end{array} \\ \begin{array}{l}\text { hydrochloride for a nerve block, it is m } \\ \text { effective than the } 1 \mathrm{Mhz} \text { that is widely } \\ \text { used in clinical situations. }\end{array}\end{array}$

$\begin{array}{ll}\begin{array}{l}\text { Administration of lidocaine } \\ \text { hydrochloride trandermally on healthy }\end{array} & \begin{array}{l}0.5 \mathrm{MHz} \text { ultrasound in phonophoresis } \\ \text { conduction anesthesia using lidocaine } \\ \text { volunteers applying } 0.5 \mathrm{MHz} \text { ULTS. }\end{array} \\ \begin{array}{l}\text { hydrochloride for a nerve block, it is mor } \\ \text { effective than the } 1 \mathrm{Mhz} \text { that is widely } \\ \text { used in clinical situations. }\end{array}\end{array}$
Kim et al.[61]

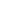

Tachibana et al.(60)
Hehn et al. [62] 
Table 2 Continued.....

\section{Anticancer drugs}

Application of a method using ULTS and nano/microbubbles to cancer gene therapy using prodrug activation therapy. Low-intensity pulsed ultrasound $\left(1 \mathrm{MHz} ; 1.3 \mathrm{~W} / \mathrm{cm}^{2}\right)$ and NBs were used to transduce the herpes simplex thymidine kinase (HSVtk) gene in vitro, leading to gene transfer.

To investigate the competitive transport across skin and back-diffusion of 5fluorouracil into coupling gel under the influence of ULTS, heat-alone and Azone ${ }^{\circledR}$ enhancement.

\section{Dramatic reductions of the tumor size by a Aoi et al.[66]} factor of four were observed.
Ultrasonication produced a decrease in percutaneous drug penetration.

Quantification studies indicated that this effect was due to the diffusive loss of the hydrophilic substance 5-fluorouracil from the skin surface into the overlying volume of coupling gel.
Meidan et al. [67]

\section{Insulin}

To determine if the $3 \times 1$ rectangular cymbal array could perform

significantly better than the $3 \times 3$ circular array for glucose reduction in hyperglycemic rabbits.

To demonstrate ultrasonic transdermal delivery of insulin in vivo using rabbits with a novel, low-profile two-by-two ULTS array based on the cymbal transducer.

The purpose of this study was to demonstrate the feasibility of ULTSmediated transdermal delivery of insulin in vivo using rats with a novel, low profile two-by-two ULTS array based on the "cymbal" transducer.
Smith et al. [70]
Lee et al.[69]

Luis et al. [68]

\author{
Lee et al. [69]
}

For the ultrasound-insulin group, the glucose level was found to decrease to $132.6 \pm 35.7 \mathrm{mg} / \mathrm{dL}$ from the initial baseline in $60 \mathrm{~min}$.

For the 60-min ULTS exposure group, the glucose level was found to decrease from the baseline to $-267.5 \pm 61.9 \mathrm{mg} / \mathrm{dL}$ in $1 \mathrm{~h}$. Moreover, to study the effects of ULTS exposure time on insulin delivery, the 20min group had essentially the same result as the 60 -min exposure at a similar intensity, which indicates that the expose time does not need to be as long for delivery.

\section{Corticosteroids}

Determination of the effect of ULTS on the transcutaneous absorption of dexamethasone when occluded with a dressing.
A phonophoretic effect occurred with dexamethasone when its application saturated the skin. 


\section{Table 2 Continued.....}

To determine if ultrasound enhances the diffusion of transdermally applied corticosteroids.

To compare the effectiveness of $0.4 \%$ Dexamethasone sodium phosphate (DEX-P) phonophoresis (PH) with $0.4 \%$ DEX-P iontophoresis (ION) therapy in the management of patients with knee joint OA.

Designing a phonophoretic drug delivery system to enhance the TA permeability and the influence of ultrasound was examined.

An acoustic model to determine the effect of ULTS on the penetration of hydrocortisone through rat skin.
The effects of phonophoresed DX can be measured in terms of reduced collagen deposition as far down as the subcutaneous tissue but not in the submuscular or subtendinous tissue.

At the end of two weeks, significant improvement in total WOMAC scores was observed in $15(60 \%)$ and $16(64 \%)$ patients in the $\mathrm{PH}$ and ION groups respectively, indicating no significant difference in the improvement rate.

The highest permeation was observed under the ultrasound treatment conditions of low frequency, high intensity, and in continuous mode.

Synergism between phonophoresis and Azone treatment was obsgerved in the enhancement of hydrocortisone percutaneous transport. It is probable that the thermal effects of ULTS were responsible for accelerated Azone diffusion through the SC.
Byl et al.[72]

Akinbo et al. [73]

Yang et al. [74]

Meidan et al. [75]

\section{Cardiotonics}

The phonophoresis of digoxin in vitro through human and hairless mouse skin.
There was no enhancement of digoxin absorption across human skin by ULTS, probably due to dermal retention of this lipophilic drug.
Machet et al.[76] McElnay et al. [77]

\section{Vasodilators}

\begin{tabular}{|c|c|c|}
\hline $\begin{array}{l}\text { Skin penetration enhancement effect of } \\
\text { ULTS (phonophoresis) on methyl } \\
\text { nicotinate in } 10 \text { healthy volunteers in a } \\
\text { double-blind, placebo-controlled, } \\
\text { crossover clinical trial. }\end{array}$ & $\begin{array}{l}\text { Ultrasound treatment applied prior to } \\
\text { methyl nicotinate led to enhanced } \\
\text { percutaneous absorption of the drug. }\end{array}$ & McElnay et al. [77] \\
\hline $\begin{array}{l}\text { Effect of permeation enhancers and } \\
\text { application of low frequency (LULTS) } \\
\text { and high frequency ultrasound } \\
\text { (HULTS) on testosterone (TS) } \\
\text { transdermal permeation after } \\
\text { application of testosterone solid lipid } \\
\text { microparticles. }\end{array}$ & $\begin{array}{l}\text { The enhancement effect of different } \\
\text { intensities of LULTS was not directly } \\
\text { proportional to the magnitude of intensity. } \\
\text { Skin exposure to HULTS or LULTS } \\
\text { before application of } 1 \% \text { DA for } 30 \text { min } \\
\text { had no superior enhancement effect over } \\
\text { application of either LULTS or HULTS } \\
\text { alone. Application of drug loaded SLM } \\
\text { offered skin protection against the irritation } \\
\text { effect produced by TS and 1\% DA. }\end{array}$ & El-Kamel et al.[78] \\
\hline
\end{tabular}


Table 2 Continued.....

\section{Cicatrizants}

The effectiveness of sonophoresis on the delivery of high molecular weight (MW) hyaluronan (HA) into synovial membrane using an animal model of osteoarthritis (OA).

\author{
Synovial fluid analysis revealed increased \\ absorption and fluorescence microscopy \\ showed deeper penetration of both \\ HA1000 and HA3000, more so with the \\ latter. \\ Park et al. [79]
}

\section{Calcein}

The skin permeation clearance $(\mathrm{Al} / \mathrm{h})$ of model hydrophilic solutes, calcein (MW 623) and-labeled dextrans [MW 4400 (FD-4) and MW 38000 (FD-40)], across the skin under the influence of ULTS was plotted against the corresponding $3 \mathrm{H}_{2} \mathrm{O}$ flux $(\mathrm{Al} / \mathrm{h})$ to estimate the potential contribution of convective solvent flow, induced by the ULTS application, to the solute transport.

Good correlations were observed between the $3 \mathrm{H}_{2} \mathrm{O}$ flux and solute clearances and, unexpectedly, the slope values obtained from linear regression of the plots were consistent for all solutes examined. Transport of intact FD-4 and FD-40 was confirmed by gel permeation chromatography. When the skin surface and deeper regions of the skin after sonophoresis of FD-40 were observed using a confocal microscope, the fluorescence of FD-40 was uniformly distributed in the area under the ULTS horn and also evident in crack-like structures in the boundary of the horn.

\section{Oligonucleotids}

To assess the potential of low frequency ULTS $\left(20 \mathrm{kHz}, 2.4 \mathrm{~W} / \mathrm{cm}^{2}\right)$ in delivering therapeutically significant quantities of anti-sense oligonucleotides into skin.

Microscopic evaluations using

fluorescently labeled oligonucleotides and sulforhodamine B revealed heterogeneous penetration into the skin. Heterogenous penetration led to the formation of localized transport pathways (LTPs), which occupied about $5 \%$ of the total exposed skin area. Immuno-histochemical studies using an oligonucleotide that reacts specifically with an antibody also confirmed penetration of ODNs into LTPs.

\section{Stimulants}

The effect of low-frequency sonophoresis on fentanyl and caffeine permeation through human and hairless rat skin was studied in vitro.
Discontinuous ULTS mode was found to be more effective in increasing transdermal penetration of fentanyl while transdermal transport of caffeine was enhanced by both continuous and pulsed mode.
Morimoto et al. [80]

Tezel et al. [81]

\section{Calcium}

\begin{tabular}{|c|c|}
\hline $\begin{array}{l}\text { Manipulation of the } \mathrm{Ca}^{2+} \text { content of the } \\
\text { upper epidermis by sonophoresis of } \\
\text { aqueous solutions containing } \\
\text { physiologic } \mathrm{Ca}^{2+}\left(\text { and } \mathrm{K}^{2+} \text { versus ion- }\right. \\
\text { free solution across hairless mouse SC. }\end{array}$ & $\begin{array}{l}\text { Sonophoresis at } 15 \mathrm{MHz} \text { did not alter } \\
\text { barrier function, but in the absence of } \mathrm{Ca}^{2+} \\
\text { the extracellular calcium content of the } \\
\text { outer epidermis, as revealed by ion capture } \\
\text { cytochemistry. }\end{array}$ \\
\hline
\end{tabular}


Table 2 Continued.....

\section{Panax notoginseng}

The phonophoretic effect of a therapeutic ULTS coupled with a Panax notoginseng (PN) gel and compared it with a therapeutic ultrasound alone for medial collateral ligament repair in rats.
This study reveals a positive ultrasonic phonophoretic effect of Panax notoginseng extract for improving the strength of ligament repair than ULTS therapy alone.
Ng et al. [84]

\section{Other applications}

i)To study the mechanisms of penetration due to ultrasound throughout the skin

\begin{abstract}
To shed light on the mechanism(s) by which low-frequency ultrasound (20 $\mathrm{KHz}$ ) enhances the permeability of the skin. The physical effects on the barrier and the transport pathway, in particular, were examined.
\end{abstract}

To investigate the effects of short time sonication of human skin at $20 \mathrm{kHz}$ and at variable intensities and duty cycles on the dynamics of fluorescein transport across the skin as well as the changes in the skin's structural integrity.

Use of quantum dots (QDs) as a tracer and confocal microscopy and transmission electron microscopy (TEM) as visualization methods, on LFS induced permeation pathways in the SC. QDs (20 nm diameter) penetrated well beyond the SC. A significant fraction (30\%) of the intercellular lipids of the SC, which are principally responsible for skin barrier function, were removed during the application of low frequency sonophoresis.
Kushner et al. [85]

Alvarez-Román et al.

[86]

A short application of ULTS enhanced the

Cancel et al. [87] transport of fluorescein across human skin by a factor in the range of 2-9 for full thickness skin samples and by a factor in the range of 2-28 000 for heat-stripped SC samples.

TEM revealed that ultrasound significantly increased the frequency of occurrence of the otherwise scattered and separated lacunar spaces in the SC. A significant increase in lacunar dimensions was observed when $1 \% \mathrm{w} / \mathrm{v}$ sodium lauryl sulfate was added to the coupling medium.

\section{ii)Tumours}

Optimization of ULTS parameters for in vivo bleomycin delivery.

To investigate if high-intensity focused ultrasound (HIFU) exposures could enhance tumor uptake of (111)In-MXB3, a murine IgG1kappa monoclonal antibody directed against the $\operatorname{Le}(\mathrm{y})$ antigen.
An effective antitumor effect was demonstrated in solid tumors of both murine and human cell lines.

The HIFU exposure shortened the peak tumor uptake time ( $24 \mathrm{vs.} 48 \mathrm{~h}$ for the control) and increased the peak tumor uptake value (38 vs. $25 \% \mathrm{ID} / \mathrm{g}$ for the control). The HIFU effect on enhancing tumor uptake was greater at earlier times up to $24 \mathrm{~h}$, but the effect was gradually diminished thereafter.
Paliwal et al. [88]
Khaibullina et al. [90]

\section{Larkin et al. [89]}

Kathes 
The changes in the mechanical and thermal hyperalgesia, nociceptive flexor reflex (NFR), as well as the swelling changes were determined.

According to the anti-hyperalgesia and antiinflammation tests, which were used to determine the change in the pain threshold, NFR and swelling showed that the group given the phonophoretic transdermal delivery of KT exhibited significantly more noticeable anti-hyperalgesic and antiinflammatory effects than those treated with the simple application of a KT gel. The transdermal application of KT gel using phonophoresis had significant anti-hyperalgesic and anti-inflammatory effects. These findings suggest that the transdermal administration of a KT gel using phonophoresis using pulsed ULTS might be useful for treating acute inflammation and pain.

To compare efficacy of pain syndrome relief in osteoarthrosis at conventional administration and ultraphonophoresis of ibuprofen (nurofen gel), Serikov et al. [56] applied ultraphonophoresis of 5\% nurofen gel to affected joints of 20 patients of the study group. The control group received only local treatment with $5 \%$ gel nurofen on the affected joints three times a day. The course of pain syndrome was assessed by the visual analogue scale (VAS). Monitoring of side effects, total blood count, blood biochemistry, urine analysis were made. They recorded significant attenuation of pain by VAS at rest, in palpation, in walking in both groups. In spite of initial pain intensification in ultraphonophoresis, positive dynamics of pain decline in the study group was more significant. Thus, their study supports anesthetic efficacy of transcutaneous $5 \%$ gel nurofen in osteoarthrosis and a rise of this efficacy in combined use of ULTS and this medicine in ultraphonophoresis.

Cabak et al. [57] examined the therapeutic effects of phonophoresis with ketoprofen in gel form in patients with enthesopathy of the elbow. Ultrasonic therapy and phonophoresis have their primary application in the physical therapy of this disorder. The main aim of Cabak et al. was to assess the effectiveness of phonophoresis. The research group consisted of 19 patients diagnosed with enthesopathy of the lateral and medial epicondyle. In the statistical analysis we included 28 elbow joints treated with phonophoresis. The effects of therapy were compared with a control group of 20 patients who were treated with only ULTS therapy.
The therapeutic series consisted of 10 treatments, using the pulse mode of ULTS and an intensity of $0.8 \mathrm{~W} / \mathrm{cm}^{2}$ in both groups. The clinical examination (objective assessment) and interview (subjective assessment) consisted of specific tests, and were separately collected. The positive effects of phonophoresis using a pharmacologically active gel with ketoprofen were shown to be highly significant in both assessments, objective and subjective. The pain symptoms in the elbow resolved in most of the patients. There were statistically significant differences between phonophoresis and ULTS therapy. Their results support the application of phonophoresis with ketoprofen in the treatment of epicondylitis.

A quantitative study of sodium diclofenac (Voltaren Emulgel, Novartis) phonophoresis was undertaken in humans by Rosim et al. [58] Fourteen healthy human volunteers were submitted to ULTS irradiation on two $225 \mathrm{~cm}^{2}$ areas on the dorsum (group A), followed by the application of the medication gel, and the plasma diclofenac mass was measured at 1, 2 and $3 \mathrm{~h}$ later by HPLC. The same procedure was repeated one month later with the same volunteers but with the ultrasound equipment switched off for the control group (group B). The plasma diclofenac mass was significantly higher in group A than in group B at $1 \mathrm{~h}(0.0987 \mu \mathrm{g} / \mathrm{mL}$ as opposed to $0.0389 \mu \mathrm{g} / \mathrm{mL})$ and $2 \mathrm{~h}(0.0724 \mu \mathrm{g} / \mathrm{mL}$ as opposed to $0.0529 \mu \mathrm{g} / \mathrm{mL})$, but not at $3 \mathrm{~h}(0.0864$ $\mu \mathrm{g} / \mathrm{mL}$ as opposed to $0.0683 \mu \mathrm{g} / \mathrm{mL}$ ). The authors conclude that previously applied therapeutic ULTS irradiation enhances the percutaneous penetration of the topical diclofenac gel, although the mechanism remains unclear.

Although topical drugs are usually applied at a convenient site, the target for the drug interaction may be systemic. The purposes of the study of Hippius et al. [59] were to investigate the in vitro penetration and the in vivo transport of flufenamic acid in dependence of ULTS. Percutaneous absorption studies were performed in various in vitro models to determine the rate of drug absorption via the skin. They designed a phonophoretic drug delivery system to investigate the influence of ULTS on transmembrane transport of different drugs. They investigated the absorption of flufenamic acid in a buffer medium in dependence of ULTS energy and application time. For evaluating membrane penetration of flufenamic acid, the concentration range of buffer solution was 
measured. Ultrasound energy was supplied for between 5 and $30 \mathrm{~min}$ at a range of intensities up to $1.5 \mathrm{~W} / \mathrm{cm}^{2}$, energy levels commonly used for therapeutic purpose. The pronounced effect of ULTS on the transmembrane absorption of the drug was observed at all ULTS energy levels studied. The time of application was found to play an important role in delivery and transport of drug. Dependent on time, they observed a rise of temperature up to 4.5 degrees $\mathrm{C}$. It appears that there was no difference between an intensity of 0.3 and $1.5 \mathrm{~W} / \mathrm{cm}^{2}$ and the measured drug concentrations in solution. The highest penetration was observed at an intensity of $1.0 \mathrm{~W} / \mathrm{cm}^{2}$ after 30 min. These results were not significantly different from concentration measurements after $30 \mathrm{~min}$ and 0.5 and $1.5 \mathrm{~W} / \mathrm{cm}^{2}$. It seems that the arise of drug concentration is caused by effects of temperature and by variation of membrane delivery in dependence of temperature. Using this in vitro model they noted it is possible to compare the transdermal penetration and absorption of commercial flufenamic ointment in volunteers.

\section{Anesthetics}

Currently available local anesthetics, such as eutectic mixture of local anesthetics (EMLA), have poor skin penetration when administrated topically. As $60 \mathrm{~min}$ is needed for EMLA to be fully effective, attempts to accelerate transdermal delivery of a local anesthetic, such as lidocaine, by use of "electrical" and "physical" energy has previously been reported. Tachibana et al. [60] determined whether ultrasonic energy can increase the local anesthetic effect of lidocaine in the skin of mice. Hairless mice were immersed in a beaker containing $2 \%$ aqueous lidocaine. ULTS $(48 \mathrm{kHz}$, $0.17 \mathrm{~W} / \mathrm{cm}^{2}$ ) was applied to the beaker by an ULTS-generating water tank for $5 \mathrm{~min}$. To examine anesthetic effects, the skin of the legs was stimulated using various voltages ranging from 0 to $50 \mathrm{~V}$ before and after treatment. The number of times the hairless mouse reacted out of six stimulations was counted. Stimulation by $15 \mathrm{~V}$ at 30 min after lidocaine and ULTS exposure resulted in positive reaction of $1.3 \pm 1.6$ compared with $5.2 \pm 2.0$ before treatment. Significant anesthetic effects continued for $2 \mathrm{~h}$. Immersion to lidocaine alone without ultrasound showed no evidence of analgesia after treatment. ULTS alone to the legs also caused no anesthetic effects. It was concluded that ULTS exposure to the legs of hairless mice along with topical $2 \%$ lidocaine solution rapidly induced an anesthetic effect.

Low-frequency ultrasound has a significant effect on the transdermal permeation of high molecular weight drugs. However, the rate of permeation in pulsed mode is quite low necessitating considerable time to apply the ULTS. $0.5 \mathrm{MHz}$ ULTS, which is a relatively higher frequency in the low-frequency range, can be applied in high intensity in continuous mode. Kim et al. [61] used a transducer to administer an anesthetic drug transdermally on healthy volunteers. The anesthetic effect was measured following administration on placebo, lidocaine $\mathrm{HCl}$ alone and lidocaine $\mathrm{HCl}$ with 0.5 and $1.0 \mathrm{MHz}$ ULTS. In surface anesthesia, the phonophoresis group showed a significantly higher pain threshold than the other groups but there was no significant difference between the phonophoresis groups according to the ULTS frequency. In conduction anesthesia, the 0.5 $\mathrm{MHz}$ phonophoresis group showed a significant change in their pain threshold and amplitude of sensory nerve action potential compared with the other groups. Although there are limitations in applying $0.5 \mathrm{MHz}$ ULTS in phonophoresis for conduction anesthesia using lidocaine hydrochloride for a nerve block, it is more effective than the $1 \mathrm{Mhz}$ that is widely used in clinical situations.

With the aid of permanent cultured MDCK (Madin Darby Canine Kidney) epithelial cell a model for investigations dealing with phonophoresis effects was developed by Hehn et al. [62]. The permeation of procaine hydrochloride through cell monolayers was examined while applying therapeutical ULTS simultaneously. It could be shown that this permeation follows Higuchi kinetics. A comparison of the velocity factors, using a continuous irradiation of 1.0 $\mathrm{W} / \mathrm{cm}^{2}$, shows about a 4.8 fold increase. Single ULTS pulses, however, result in a short-time enhancement of the permeation. The conclusion can be drawn that extent and velocity of the permeation of procaine hydrochloride through MDCK monolayer can be controlled by phonophoresis

\section{Antibiotics}

Liu et al. [63] investigated the topical transport of 
Cyclosporin A using low-frequency ULTS throughout rat skin. Studies of intensity and exposure time acting on the deposition of Cyclosporin A into deeper skin of in vitro sonophoresis were performed. Low-frequency ULTS increased the amount of Cyclosporin A retained in the skin only seven times than the passive diffusion. Furthermore, they also tested the synergistic effect of ULTS and other approaches such as chemical enhancers and electroporation on topical drug delivery of Cyclosporin A. They found that the efficacy of low-frequency ULTS in enhancing topical delivery could be further increased by pretreatment of skin with chemical enhancers, such as Azone ${ }^{\circledR}$ and sodium lauryl sulfate (SLS). Meanwhile only a small amount was seen to across the full skin into the receiver compartment. Trimodality treatment comprising of pretreatment with Azone ${ }^{\circledR}+$ ULTS in combination followed by electroporation was not effective in enhancing the topical delivery of Cyclosporin A. However, this combination strategy increased the penetration of Cyclosporin.A through rat skin by order of 15. In general, the enhanced skin accumulation of Cyclosporin A by the combination of low-frequency ULTS and chemical enhancers could help significantly to optimize the targeting of the drug without of a concomitant increase of the systemic side effects.

Santoianni et al. [64] evaluated the efficacy of low frequency sonophoresis (LFS) at $25 \mathrm{KHz}$ produced by a sonicator apparatus for treatment of alopecia areata, melasma and solar lentigo. Thirty patients affected by alopecia areata were treated by application of methylprednisolone or cyclosporine solution followed by LFS. In a case-control study 48 women with melasma and 48 with solar lentigo were also treated by depigmenting emulsion and LFS application. For alopecia areata after 36 applications with LFS and 3-month treatment the results were: 57 percent partial regrowth and 29 percent total with methylprednisolone; and 33 percent partial regrowth and 34 percent total when cyclosporine was used. For melasma and solar lentigo the results when the drug application was followed by LFS, were after 3-month and twice a week application: 75 percent complete depigmentation and 25 percent partial for melasma, 43 percent total regression and 57 percent partial for solar lentigo. This was the first report of sonophoresis at a frequency of $25 \mathrm{KHz}$ in dermatocosmetology. The study showed that LFS, a not aggressive technique, enhanced penetration of topic agents obtaining effects at the level of the epidermis, dermis and appendages (intradermal delivery), giving better results in the treatment of some cosmetic skin disorders.

Ragelis et al. [65] performed a total of 451 experiments (130 with the method of electrophoresis and 321 with the method of phonophoresis) on healthy rabbits of the same species, age, weight and sex with the use of the galvanization apparatus "Potok-1" and ULTS therapeutic apparatus "VTP-1". The penetration levels of tetracycline into the tissues after its administration with the modified methods of electro- and phonophoresis were determined. The dependence of the process on the current density, ULTS intensity, time and antibiotic concentration was studied. The efficacy of the routine electro- and phonophoresis methods was compared with that of the modified ones. It was found that the tissue levels of tetracycline administered with the modified methods of electro- and phonophoresis increased with an increase in the current density or ULTS intensity, the procedure time and antibiotic concentration. When tetracycline was administered with the modified method of electrophoresis its levels were highest in the skin, lower in the muscles and minimum in the bones. With the use of the modified method of phonophoresis the highest levels were in the skin, the lowest in the bones and the minimum in the muscles. The tissue levels of tetracycline administered with the modified methods of electro- and phonophoresis were higher than those obtained with the routine methods of electro- and phonophoresis.

\section{Anticancer drugs}

Aoi et al. [66] evaluated the application of a method using ULTS and nano/microbubbles to cancer gene therapy using prodrug activation therapy. Lowintensity pulsed ULTS ( $\left.1 \mathrm{MHz} ; 1.3 \mathrm{~W} / \mathrm{cm}^{2}\right)$ and NBs were used to transduce the herpes simplex thymidine kinase (HSVtk) gene in vitro, leading to gene transfer. The addition of ganciclovir (GCV) to the transduced cells led to HSVtk/GCV-dependent cell death mediated by apoptosis. This technology was then assessed in vivo, using mice bearing subcutaneous tumors $\left(1 \mathrm{MHz} ; 3.0 \mathrm{~W} / \mathrm{cm}^{2}\right)$. Gene transfer to the tumor, measured by luciferase 
activity, was transient, with a peak of expression 24 $\mathrm{h}$ after transduction, and decreased at $48 \mathrm{~h}$, demonstrating the transient nature of ULTS/NBmediated gene transfer. The therapeutic potential of this approach was evaluated through repeated intratumoral gene delivery using ULTS/NBmediated transfer of the HSVtk gene, followed by recurrent administration of GCV, using two different experimental treatment protocols. In both cases, dramatic reductions of the tumor size by a factor of four were observed. Altogether, these data demonstrate the potential of ULTS/NB as a new physical gene delivery method for cancer gene therapy.

Meidan et al. [67] investigated the competitive transport across skin and back-diffusion of 5-fluorouracil into coupling gel under the influence of ULTS, heat-alone and Azone $^{\circledR}$ enhancement. The ULTS effect on 5-fluorouracil penetration through whole rat skin was investigated in modified diffusion cells using a commercial ULTS generator which was calibrated with a bilaminar membrane hydrophone. Ultrasonic dosimetry measurements demonstrated that the skin membrane was subjected to a complex and unpredictable standing wave field which induced physiologically acceptable heating of the tissue. Surprisingly, ultrasonication produced a decrease in percutaneous drug penetration. Quantification studies indicated that this effect was due to the diffusive loss of the hydrophilic substance 5fluorouracil from the skin surface into the overlying volume of coupling gel. This phenomenon could be duplicated by the application of conductive heating, indicating that the thermal effects of ULTS were probably responsible for accelerated 5-fluorouracil diffusion through the gel. This study acutely demonstrates how formulation design of the donor vehicle/coupling gel may radically affect therapeutic efficacy in phonophoretic systems.

\section{Insulin}

Circular cymbal ULTS arrays have been shown to be effective in delivering therapeutic levels of insulin in rats, rabbits, and pigs. To improve delivery efficiency, a rectangular cymbal design was desired in order to achieve a broader spatial intensity field without increasing the size of the device or the spatial-peak temporal-peak intensity. Luis et al. [68] with a similar intensity $\left(50 \mathrm{mWcm}^{-}\right.$
${ }^{2}$ ), determined if the $3 \times 1$ rectangular cymbal array could perform significantly better than the $3 \times 3$ circular array for glucose reduction in hyperglycemic rabbits. Rabbit experiments were performed using three groups: nonsonicated control, ULTS exposure using a circular cymbal array, and ULTS exposure using a rectangular cymbal array. Rabbits were anesthetized and a water tight reservoir that held the insulin was fastened on the rabbit's thigh. At the beginning of the experiment and every $15 \mathrm{~min}$ for $90 \mathrm{~min}$, the blood glucose level was determined. For comparison between individual rabbits, the absolute level is normalized by subtracting out the baseline in order to arrive at the change in glucose level. For the control group, the normalized glucose level increased (more hyperglycemic) to $+80.0 \pm 28.8 \mathrm{mg} / \mathrm{dL}$. Using the circular array, the glucose level decreased to $146.7 \pm 17.8 \mathrm{mgdl}$ at $90 \mathrm{~min}$. However, using the rectangular cymbal array, the glucose decreased faster and to a level of $-200.8 \pm 5.9 \mathrm{mgdl}$ after 90 min. These results indicated the feasibility of the rectangular cymbal array as an improved device for drug delivery.

Recent studies have shown that ULTSmediated transdermal drug delivery offers a promising potential for noninvasive drug administration. The purpose of Lee et al. [69] was to demonstrate ultrasonic transdermal delivery of insulin in vivo using rabbits with a novel, lowprofile two-by-two ULTS array based on the cymbal transducer. As a practical device, the cymbal array $(\mathrm{f}=20 \mathrm{kHz})$ was $37 \times 37 \times 7 \mathrm{~mm}^{3}$ in size and weighed less than $22 \mathrm{~g}$. Using the same array on hyperglycemic rats, their previous experiments demonstrated that blood glucose would decrease $233.3 \pm 22.2 \mathrm{mg} / \mathrm{dL}$ in $90 \mathrm{~min}$ from $5 \mathrm{~min}$ of pulsed ULTS exposure. With a similar intensity $\left(\right.$ Isptp $=100 \mathrm{~mW} / \mathrm{cm}^{2}, 20 \%$ duty cycle), their goal was to determine if the same effect could be achieved with rabbits. Experiments were performed in New Zealand white rabbits divided into three groups: two controls and one ULTS with insulin exposure. At the beginning of the experiment and every $15 \mathrm{~min}$ for $90 \mathrm{~min}, 0.3 \mathrm{~mL}$ of blood was collected from the ear vein to determine the blood glucose level using a glucose monitoring system. For both controls, insulin-no ULTS and salineULTS, the blood glucose level varied from the initial baseline by approximately $75 \mathrm{mg} / \mathrm{dL}$. However, for the ULTS-insulin group, the glucose 
level was found to decrease to $-132.6 \pm 35.7 \mathrm{mg} / \mathrm{dL}$ from the initial baseline in $60 \mathrm{~min}$. Even after the array and insulin reservoir were removed, the blood glucose level of ultrasound-insulin group continued to decrease to $-208.1 \pm 29 \mathrm{mg} / \mathrm{dL}$ from the initial baseline. These results indicate the feasibility of using a low-cost, lightweight cymbal array for enhanced transdermal insulin delivery using ULTS.

Smith et al. [70] demonstrated the feasibility of ULTS-mediated transdermal delivery of insulin in vivo using rats with a novel, low profile two-by-two ULTS array based on the "cymbal" transducer. As a practical device, the cymbal array ( $\mathrm{f}=20 \mathrm{kHz}$ ) was $37 \times 37 \times 7 \mathrm{~mm}$ in size, and weighed less than $22 \mathrm{~g}$. A total of 20 SpragueDawley rats (350 to $450 \mathrm{~g}$ ) were divided into four groups, two controls and two ULTS exposures, with five rats in each group. The rats were anesthetized and shaved; a water-tight standoff reservoir, which held the insulin or saline, was sealed against the rat's abdomen and the ULTS array. At the beginning of the experiment and every $30 \mathrm{~min}$ for $90 \mathrm{~min}, 0.3 \mathrm{~mL}$ of blood was collected from the jugular vein to determine the blood glucose level. For comparison between the rats, the change in the glucose level for each rat was normalized to a baseline (i.e., $0 \mathrm{mg} / \mathrm{dL}$ ). The first control group used insulin in the reservoir with no ULTS and the second control group had saline in the reservoir with ULTS operating at I(SPTP) $=100 \mathrm{~mW} / \mathrm{cm}^{2}$ for $60 \mathrm{~min}$. For the experiments, the third group employed insulin with ULTS exposure for $60 \mathrm{~min}$ $\left(\mathrm{I}(\mathrm{SPTP})=100 \mathrm{~mW} / \mathrm{cm}^{2}\right)$, whereas the last group used insulin with ULTS operating with a 20 -min exposure $\left(\mathrm{I}(\mathrm{SPTP})=100 \mathrm{~mW} / \mathrm{cm}^{2}\right)$ to examine the effects of time on delivery. For the 60-min ULTS exposure group, the glucose level was found to decrease from the baseline to $-267.5 \pm 61.9 \mathrm{mg} / \mathrm{dL}$ in $1 \mathrm{~h}$. Moreover, to study the effects of ULTS exposure time on insulin delivery, the 20-min group had essentially the same result as the 60-min exposure at a similar intensity, which indicates that the expose time does not need to be as long for delivery.

\section{Corticosteroids}

Saliba et al. [71] found that a phonophoretic effect occurred with dexamethasone when its application saturated the skin. 2-way repeated-measures analysis of variance revealed a significant main effect for ULTS treatment. The rate of appearance and the total concentration of dexamethasone in the serum were greater in subjects after phonophoresis than after sham ULTS. The sham group had only trace amounts of dexamethasone in the serum, indicating that drug absorption was negligible without the ULTS energy. The effect size of the phonophoresis condition fell within a $95 \%$ confidence interval after the baseline measurement.

Although physical therapists and physicians often treat patients with local musculoskeletal inflammation using topically applied steroids enhanced with ULTS, there is a paucity of research confirming that phonophoresis significantly enhances drug diffusion. The purpose of Byl et al. [72] was to determine if ULTS enhances the diffusion of transdermally applied corticosteroids. Diffusion was measured secondarily in terms of collagen deposition [estimated by levels of hydroxyproline in polytetrafluroethylene (ePTFE) tubing] and cellular activity (measured by levels of DNA). Sixteen pieces of ePTFE tubing were subcutaneously implanted on the dorsum of five mini Yucatan pigs. Pairs of tubing were randomly assigned to sham control or treatment groups. Over the paired ePTFE tubes in the treatment groups, a single transdermal application of hydrocortisone acetate (HC) or dexamethasone (DX) was applied to the skin by rubbing, sonating with the drug mixed in the acoustic gel $\left(1.5 \mathrm{~W} / \mathrm{cm}^{2}, 1 \mathrm{MHz}, 5\right.$ minutes), or injecting the drug into the tubing. Four additional ePTFE tubes were threaded in the extremities, two submuscularly and two subtendinously, with random assignment to a sham control or a DX sonation treatment group. At the end of a week, the mean hydroxyproline levels in the swine were lower than expected $($ mean $=9.3 \mu \mathrm{g} / \mathrm{cm}$ compared to an expected mean $=22.2 \mu \mathrm{g} / \mathrm{cm})$. Comparing the control and skin-applied groups with the injected and sonated treatment groups, the hydroxyproline was found to be $50 \%$ lower in the DX-injected, DXsonated, and HC-injected sites. However, statistically there were no significant differences in DNA or hydroxyproline levels between the HC subcutaneous control and treatment groups or the DX submuscular and subtendinous groups. There was a significant main effect of group on hydroxyproline levels in the group of DX-treated, subcutaneously implanted ePTFE tubes. Post hoc testing revealed a significant difference between the skin-rubbed and control groups together compared 
to the DX-injected and DX-sonated groups together. These findings indicate that the effects of phonophoresed DX can be measured in terms of reduced collagen deposition as far down as the subcutaneous tissue but not in the submuscular or subtendinous tissue. However, a single application may not have a measurable effect on cellular activity after 7 days of healing. The unusually low level of hydroxyproline across all groups suggests that phonophoresis with steroids may have had a systemic as well as a local effect.

Many treatment options, including nonpharmacological and pharmacological measures, have been recommended in the management of osteoarthritis (OA). Among the nonpharmacological approach is physiotherapy, which involves the use of physical modalities like, heat therapy, exercise therapy, electrical stimulation, therapeutic ULTS, iontophoresis, and phonophoresis. The study of Akinbo et al. [73] was designed to compare the effectiveness of $0.4 \%$ Dexamethasone sodium phosphate (DEX-P) phonophoresis (PH) with $0.4 \%$ DEX-P iontophoresis (ION) therapy in the management of patients with knee joint OA. Fifty patients with a mean age of $53.6 \pm 8.9$ years were randomly assigned to $\mathrm{PH}$ or ION groups with 25 patients in each group. ULTS waves of $1 \mathrm{MHz}$ frequency was applied for 5 minutes to the target knee, so also was the direct current for 10 minutes for 10 sessions treatment period. Western Ontario and McMaster University Osteoarthritis Index (WOMAC) scores, 20 meters ambulatory time, and knee range of motion (ROM) were evaluated before and after therapy as the outcome measures.

At the end of two weeks, significant improvement in total WOMAC scores was observed in $15(60 \%)$ and $16(64 \%)$ patients in the $\mathrm{PH}$ and ION groups respectively, indicating no significant difference in the improvement rate. Twenty metres ambulatory time and knee range of motion also improved significantly in both groups, yet these variables showed no significant difference between the two groups. Both therapeutic modalities were found to be effective and generally well tolerated after 10 treatment sessions. DEX-P phonophoresis was not superior to DEX-P iontophoresis in the treatment of patients with OA of the knee.

Triamcinolone acetonide (TA) is a corticosteroid that is used in the systemic and topical treatment of many inflammatory diseases. In this study, Yang et al. [74] designed a phonophoretic drug delivery system to enhance the TA permeability and the influence of ULTS was examined. In order to establish the transdermal delivery system for TA, a hydrophilic carbopol gel containing TA was prepared after adopting phonophoresis. A permeation study through mouse skin was performed at 37 degrees $C$ using a Franz diffusion cell, and the ULTS treatment was carried out for $10 \mathrm{~h}$. The level of TA permeation through the skin was evaluated under various ULTS conditions including the frequency $(1.0,3.0 \mathrm{MHz})$, intensity $\left(1.0,2.5 \mathrm{~W} / \mathrm{cm}^{2}\right)$, and duty cycle (continuous, pulse mode) using a $0.5 \% \mathrm{TA}$ gel. The highest permeation was observed under the ULTS treatment conditions of low frequency, high intensity, and in continuous mode.

The aim of the study of Meidan et al. [75] was to define an acoustic model to determine the effect of ULTS on the penetration of hydrocortisone through whole rat skin. ULTS dosimetry measurements were used to define an ULTS source used to measure the phonophoretic enhancement of hydrocortisone transport through rat skin in vitro. The effect of conductive heating was also studied. Acoustic dosimetry measurements indicated that the skin barrier was exposed to ULTS standing waves and this focused heat generation within the tissue. While sonication alone did not significantly enhance hydrocortisone permeation, a significant synergistic effect was observed with Azone ${ }^{\mathbb{B}}$ but not with oleic acid. The ultrasound-Azone ${ }^{\circledR}$ effect could be duplicated with conductive heating.

Synergism between phonophoresis and Azone $^{\circledR}$ treatment was observed in the enhancement of hydrocortisone percutaneous transport. It is probable that the thermal effects of ULTS were responsible for accelerated Azone ${ }^{\circledR}$ diffusion through the SC.

\section{Cardiotonics}

Machet et al. [76] studied the phonophoresis of digoxin in vitro through human and hairless mouse skin. Sonication was carried out with continuous mode at an intensity of 1 and $3 \mathrm{~W} / \mathrm{cm}^{2}$ and a frequency of $3.3 \mathrm{MHz}$ for $10 \mathrm{~min}$. Sonication at $3 \mathrm{~W} / \mathrm{cm}^{2}$ significantly increased the absorption of digoxin through mouse skin. Percutaneous penetration was not increased using an intensity of 
$1 \mathrm{~W} / \mathrm{cm}^{2}$ under the same experimental conditions. Enhanced digoxin penetration at $3 \mathrm{~W} / \mathrm{cm}^{2}$ can be explained by the mechanical and/or thermal action of ULTS waves. Thermal simulation from electrical resistance increased digoxin flux in comparable amounts to those obtained by sonication at $3 \mathrm{~W} / \mathrm{cm}$ ${ }^{2}$. There was no enhancement of digoxin absorption across human skin by ULTS, probably due to dermal retention of this lipophilic drug.

\section{Vasodilators}

McElnay et al. [77] investigated the skin penetration enhancement effect of ULTS (phonophoresis) on methyl nicotinate in 10 healthy volunteers in a double-blind, placebo-controlled, crossover clinical trial. Each treatment consisted of the application of ULTS massage $(3.0 \mathrm{MHz}, 1.0$ $\mathrm{W} / \mathrm{cm}^{2}$ continuous output) or placebo massage (0 $\mathrm{MHz}$ ) for $5 \mathrm{~min}$ to the forearms of the volunteers, followed by a standardized application of methyl nicotinate at intervals of $15 \mathrm{sec}, 1 \mathrm{~min}$, and $2 \mathrm{~min}$ post-massage. Percutaneous absorption of methyl nicotinate was monitored using laser Doppler velocimetry. ULTS treatment applied prior to methyl nicotinate led to enhanced percutaneous absorption of the drug, for example, ULTS treatment data versus control data at 2 min showed significant increases in the peak blood flow (125.8 \pm 12.0 vs $75.3 \pm 10.4 \%$ flux) and in the AUC for blood flow $(2630.3 \pm 387.5$ vs $1567.6 \pm 183.5 \%$ flux.min). The results of this study suggest that ULTS affects the skin structure to provide skin penetration enhancement. This finding was consistent with the proposed hypothesis that phonophoresis acts by disordering the structured lipids in the SC.

\section{Hormones}

El-Kamel et al. [78] investigated the effect of permeation enhancers and application of low frequency (LULTS) and high frequency ultrasound (HULTS) on testosterone (TS) transdermal permeation after application of testosterone solid lipid microparticles (SLM). SLM formulations contained $10 \%$ compritol and $5 \mathrm{mg}$ TS /g of SLM. The permeation experiments were performed using Franz diffusion cells and abdominal rat skin. The examined permeation enhancers were $1 \%$ oleic acid (OAc) or $1 \%$ dodecylamine (DA). HULTS (1
$\mathrm{MHz}$ ) was applied in a continuous mode for $1 \mathrm{~h}$ at intensity $0.5 \mathrm{~W} / \mathrm{cm}^{2}$. Different intensities and application time of pulsed LULTS $(20 \mathrm{kHz})$ were also examined. Additionally, the effect of combination of ULTS and OA or DA was investigated. Skin irritation and histological changes were also evaluated. The results revealed that SLMs have an occlusive effect on the skin. Statistical analysis revealed the following order for the permeation of TS: 1\% DA for $30 \mathrm{~min}>$ HULTS $+1 \%$ DA for $30 \mathrm{~min}=$ HULTS=HULTS + SLM containing $1 \% \quad \mathrm{OA}>\mathrm{SLM}$ containing $1 \%$ $\mathrm{OA}=$ control. At total application time of LULTS 6, 12 , and $15 \mathrm{~min}$ the flux increased by $1.86,4.63$, and 4.77 fold, respectively. The enhancement effect of different intensities of LULTS was not directly proportional to the magnitude of intensity. Skin exposure to HULTS or LULTS before application of $1 \%$ DA for $30 \mathrm{~min}$ had no superior enhancement effect over application of either LULTS or HULTS alone. Application of drug loaded SLM offered skin protection against the irritation effect produced by TS and 1\% DA. Histological characteristics of the skin were affected to various extents by application of enhancers or ultrasound. In general, application of LULTS gave higher TS permeation than HULTS. However, safe application of LULTS should be practiced by careful selection of exposure parameters.

\section{Cicatrizants}

Park et al. [79] determined the effectiveness of sonophoresis on the delivery of high molecular weight (MW) hyaluronan (HA) into synovial membrane using an animal model of osteoarthritis (OA). A total of $1000 \mathrm{kDa}$ (HA1000) and $3000 \mathrm{kDa}$ (HA3000) HA were labeled with fluorescein and injected into the knees of rabbits. Low-intensity continuous ULTS at $1 \mathrm{MHz}, 400 \mathrm{~mW} / \mathrm{cm}^{2}$ was applied to the knees for $10 \mathrm{~min}$ treatment bid. Synovial fluid analysis revealed increased absorption and fluorescence microscopy showed deeper penetration of both HA1000 and HA3000, more so with the latter. Histologic examination indicated that ULTS treatment resulted in no apparent damage to the synovial membrane. These results suggested that simultaneous sonication with HA injection might compensate for the short halflife of HA. Consequently, this dual treatment would 
render HA a far more effective tool in the management of OA.

\section{Calcein}

Morimoto et al. [80] examined a relationship between hydrophilic solute and water (vehicle) transports in the excised hairless rat skin in the presence of ULTS $\left(41 \mathrm{kHz}, 60-300 \mathrm{~mW} / \mathrm{cm}^{2}\right)$ irradiation and also conducted skin surface observation using confocal microscopy. When the applied intensity was increased stepwise over the rage of $60-300 \mathrm{~mW} / \mathrm{cm}^{2}$, the transport of tritiated water $\left(3 \mathrm{H}_{2} \mathrm{O}\right)$ was increased 140 -fold in an intensity-dependent manner and this returned to normal on stopping the ULTS application. The skin permeation clearance $(\mathrm{Al} / \mathrm{h})$ of model hydrophilic solutes, calcein (MW 623) and-labeled dextrans [MW 4400 (FD-4) and MW 38000 (FD-40)], across the skin under the influence of ULTS was plotted against the corresponding $3 \mathrm{H}_{2} \mathrm{O}$ flux $(\mathrm{Al} / \mathrm{h})$ to estimate the potential contribution of convective solvent flow, induced by the ULTS application, to the solute transport. Good correlations were observed between the $3 \mathrm{H}_{2} \mathrm{O}$ flux and solute clearances and, unexpectedly, the slope values obtained from linear regression of the plots were consistent for all solutes examined. Transport of intact FD-4 and FD-40 was confirmed by gel permeation chromatography. When the skin surface and deeper regions of the skin after sonophoresis of FD-40 were observed using a confocal microscope, the fluorescence of FD-40 was uniformly distributed in the area under the ULTS horn and also evident in crack-like structures in the boundary of the horn. On the other hand, a hexagonal structure of horny cells in the SC observed by poststaining with rhodamine B was fully conserved in the area under the horn. These findings suggest that $41 \mathrm{kHz}$ ultrasound can increase the transdermal transport of hydrophilic solutes by inducing convective solvent flow probably via both corneocytes and SC lipids as well as newly developed routes. Their observation also suggests that $41 \mathrm{kHz}$ ULTS has the potential to deliver hydrophilic large molecules transdermally.

\section{Oligonucleotids}

Topical delivery of oligonucleotides, though attractive for the treatment of skin disorders, is limited by the low permeability of the SC. Tezel et al. [81] assessed the potential of low frequency ULTS $(20 \mathrm{kHz}, 2.4 \mathrm{~W} / \mathrm{cm} 2)$ in delivering therapeutically significant quantities of anti-sense oligonucleotides into skin. Estimated concentrations of oligonucleotides (ODNs) in the superficial layers of the skin ranged from $0.5 \%$ to $5 \%$ of the donor concentration after a 10-min application of ULTS and ODN. Microscopic evaluations using fluorescently labeled oligonucleotides and sulforhodamine B revealed heterogeneous penetration into the skin. Heterogenous penetration led to the formation of localized transport pathways (LTPs), which occupied about $5 \%$ of the total exposed skin area. Immuno-histochemical studies using an oligonucleotide that reacts specifically with an antibody also confirmed penetration of ODNs into LTPs. Histologic studies revealed that no gross structural changes were induced in the skin due to ULTS application. These results show successful delivery of anti-sense oligonucleotides using low-frequency ULTS.

\section{Stimulants}

The effect of low-frequency sonophoresis on fentanyl and caffeine permeation through human and hairless rat skin was studied in vitro by Boucaud et al. [82]. Experiments were performed using $20 \mathrm{kHz}$ ULTS applied at either continuous or discontinuous mode and with an average intensity of $2.5 \mathrm{~W} / \mathrm{cm}^{2}$. The results showed that lowfrequency ULTS enhanced the transdermal transport of both fentanyl and caffeine across human and hairless rat skin. This was explained by both increasing flux during sonication and shortening the lag time. Discontinuous mode was found to be more effective in increasing transdermal penetration of fentanyl while transdermal transport of caffeine was enhanced by both continuous and pulsed mode. Histological and electron microscopy studies showed that human and hairless rat skin was unaffected by ULTS exposure. Further studies will be necessary to determine the relative contribution of ULTS parameters in low-frequency ULTSinduced percutaneous enhancement of drug transport. 


\section{Calcium}

Menon et al. [83] manipulated the $\mathrm{Ca}^{2+}$ content of the upper epidermis by sonophoresis of aqueous solutions containing physiologic $\mathrm{C}^{2+}$ (and $\mathrm{K}^{2+}$ ) versus ion-free solution across hairless mouse SC. Sonophoresis at $15 \mathrm{MHz}$ did not alter barrier function, but in the absence of $\mathrm{Ca}^{2+}$ the extracellular calcium content of the outer epidermis, as revealed by ion capture cytochemistry, was displaced downward the basal layer and dermis. In contrast, following sonophoresis of $\mathrm{Ca}^{2+-}$ containing solutions, the extracellular $\mathrm{Ca}^{2+}$ gradient became obscured by excess $\mathrm{Ca}^{2+}$ in the cytosol at all levels of the epidermis. These results demonstrated that the epidermal extracellular calcium content in the upper epidermis can be manipulated by sonophoresis without prior barrier disruption, and that changes in the $\mathrm{Ca}^{2+}$ gradient induce lamellar body secretion, independent of barrier disruption

\section{Panax notoginseng}

$\mathrm{Ng}$ et al. [84] examined the phonophoretic effect of a therapeutic ULTS coupled with a Panax notoginseng (PN) gel and compared it with a therapeutic ULTS alone for medial collateral ligament repair in rats. Twenty mature male Sprague-Dawley rats receiving surgical transection to the left medial collateral ligament (MCL) were divided randomly into three groups: ULTS, ULTS with PN coupling gel and control. The treatments started on day 3 after surgery for six days per week over a two-week period. The ULTS group received 4 min of pulse ULTS $(1 \mathrm{MHz})$ at the intensity of $0.5 \mathrm{~W} / \mathrm{cm}^{2}$ with a normal ultrasonic coupling gel. The PNULTS group received the same ultrasound treatment, but with a coupling gel that contained PN extract. The control group received a placebo ULTS treatment similar to the other two groups. On day 17 , the ligaments were mechanically tested for loadrelaxation, stiffness and ultimate tensile strength (UTS). Values of the left side were normalized against that of the right side of each animal for analysis. Results revealed significantly higher normalized stiffness and UTS in the PNULTS group than the other two groups, but insignificant difference in load-relaxation among all groups. This study reveals a positive ultrasonic phonophoretic effect of Panax notoginseng extract for improving the strength of ligament repair than ULTS therapy alone.

Other uses

To study the mechanisms of penetration due to ultrasound throughout the skin

Recent advances in low-frequency sonophoresis have focused on the existence of hypothesized localized transport regions (LTRs). However, there has been no actual experimental demonstration that the hypothesized LTRs are, in fact, localized regions of high permeability. Through a series of low-frequency sonophoresis experiments conducted with full-thickness pig skin, in the presence of the surfactant sodium lauryl sulfate (SLS), in which we have separately measured the transport of calcein through the LTRs, which have areas ranging from 10 to $40 \mathrm{~mm}^{2}$, and the surrounding regions of the skin (the non-LTRs) by means of a novel masking technique, Kushner IV et al. [85] demonstrated that the calcein permeability through the LTRs is approximately 80-fold higher than the calcein permeability through the non-LTRs, suggesting that the LTRs are structurally perturbed to a greater extent than the non-LTRs from the exposure to the ultrasound/SLS system. In addition, we propose basic models to predict the total skin transdermal permeability from the transdermal permeabilities of the LTRs and the non- LTRs, and then compare the predictions to the experimental data obtained from the masking experiments. They also demonstrated that both the LTRs and the non-LTRs exhibit significant decreases in skin electrical resistivity relative to untreated skin ( $\sim 5000$-fold and $\sim 170$ fold, respectively), suggesting the existence of two levels of significant skin structural perturbation due to ULTS exposure in the presence of SLS. Finally, an analysis of the porosity/tortuosity ratio $(€ / t)$ values suggests that trans-cellular transdermal transport pathways are present within the highly permeable, and highly structurally perturbed, LTRs.

Alvarez-Román et al. [86] sheded light on the mechanism(s) by which low-frequency ULTS $(20 \mathrm{KHz})$ enhances the permeability of the skin. The physical effects on the barrier and the transport pathway, in particular, were examined. The amount of lipid removed from the intercellular domains of the SC following sonophoresis was determined by infrared spectroscopy. Transport of the fluorescent probes nile red and calcein, under the influence of 
ULTS, was evaluated by laser scanning confocal microscopy. The results were compared with the appropriate passive control data and with data obtained from experiments in which the skin was exposed simply to the thermal effects induced by ULTS treatment. A significant fraction (30\%) of the intercellular lipids of the $\mathrm{SC}$, which are principally responsible for skin barrier function, were removed during the application of low frequency sonophoresis. Although the confocal images from the nile red experiments were not particularly informative, ULTS clearly and significantly (again, relative to the corresponding controls) facilitated transport of the hydrophilic calcein via discrete permeabilized regions, whereas other areas of the barrier were apparently unaffected.

Lipid removal from the SC is implicated as a factor contributing the observed permeation enhancement effects of low-frequency ULTS. However, microscopic observations imply that sonophoresis induces localized permeation pathways at discrete sites.

Cancel et al. [87] investigated the effects of short time sonication of human skin at $20 \mathrm{kHz}$ and at variable intensities and duty cycles on the dynamics of fluorescein transport across the skin as well as the changes in the skin's structural integrity. They found that a short application of ULTS enhanced the transport of fluorescein across human skin by a factor in the range of 2-9 for full thickness skin samples and by a factor in the range of 2-28 000 for heat-stripped SC samples The electrical resistance of the skin decreased by an average of $20 \%$ for full thickness samples and $58 \%$ for SC samples. Increasing the duty cycle from 10 to $60 \%$ caused a significant increase in permeability enhancement from 2.3 to 9.1 , and an increase in intensity from 8 to $23 \mathrm{mWcm}^{-2}$ induced a significant increase in permeability enhancement from 2 to 7.4, indicating a clear dependence of the permeability on both duty cycle and intensity. The increase in solute flux upon ULTS exposure was immediate, demonstrating for the first time the fast response dynamics of sonophoretic enhancement.

In addition, a quantitative analysis of the thermal and convective dispersion effects associated with ULTS application showed that each contributes significantly to the overall permeability enhancement observed.

Low-frequency sonophoresis (LFS) has been well documented to enhance the permeability of skin to macromolecular drugs via induction of localized transport regions. However, the organizational details of epidermis, specifically SC, during sonophoresis are beyond the resolution limit of common histo-optical microscopy tools, which fail to reveal any notable structural alterations in these regions at a submicroscopic scale. Paliwal et al. [88] used quantum dots (QDs) as a tracer and confocal microscopy and transmission electron microscopy (TEM) (with $\mathrm{OsO} 4$ and $\mathrm{RuO} 4$ postfixation) as visualization methods, on LFS induced permeation pathways in the SC. QDs $(20 \mathrm{~nm}$ diameter) penetrated well beyond the SC. TEM revealed that ultrasound significantly increased the frequency of occurrence of the otherwise scattered and separated lacunar spaces in the SC. A significant increase in lacunar dimensions was observed when $1 \% \mathrm{w} / \mathrm{v}$ sodium lauryl sulfate was added to the coupling medium. These studies show that LFS induces dilatation and higher connectivity of voids in the SC, possibly leading to formation of a three-dimensional porous network, which is capable of transporting QDs as well as macromolecules across the SC. This contention is consistent with previously conceived theoretical mechanistic understanding of LFS-induced enhanced transport across the skin.

Tumors

Bleomycin is a nonpermeant, hydrophilic macromolecule with a high intrinsic anticancer cytotoxicity. However, the cytotoxic potential of the drug is restricted by its low membrane permeability. Application of low-intensity ULTS to growing tumors enhances intracellular delivery of bleomycin after IP or intratumoral administration, thereby potentiating its cytotoxicity. Optimization of ULTS parameters for in-vivo bleomycin delivery was undertaken by Larkin et al. [89] and an effective antitumor effect was demonstrated in solid tumors of both murine and human cell lines. Cell death after treatment was shown to occur by an apoptotic mechanism. The results achieved in these experiments were equivalent to those achieved using electroporation to mediate delivery of bleomycin-electrochemotherapy. They found that, although temperature rises of up to 5 degrees $\mathrm{C}$ occur using the optimized ULTS conditions, this effect is not responsible for the potentiated drug cytotoxicity. This technique could be used with 
focused ULTS or with endoscopic ULTS probes to develop a localized and effective anticancer treatment with little or no systemic toxicity.

Khaibullina et al. [90] determined if pulsed high-intensity focused ultrasound (HIFU) exposures could enhance tumor uptake of (111)In-MX-B3, a murine IgG1kappa monoclonal antibody directed against the Le(y) antigen. MX-B3 was labeled with (111)In, purified, and confirmed for its binding to the antigen-positive A431 cell line. Groups of nude mice were inoculated subcutaneously with A431 tumor cells on both hind flanks. A tumor on one flank was treated with pulsed-HIFU; the other tumor was used as an untreated control. Within 10 min after the HIFU exposure, the mice received intravenous (111)In-MX-B3 for imaging and biodistribution studies. Mice were euthanized at 1 , 24,48 , and $120 \mathrm{~h}$ after injection for biodistribution studies. The HIFU exposure shortened the peak tumor uptake time ( $24 \mathrm{vs} .48 \mathrm{~h}$ for the control) and increased the peak tumor uptake value ( 38 vs. 25 $\% \mathrm{ID} / \mathrm{g}$ [percentage injected dose per gram] for the control). The HIFU effect on enhancing tumor uptake was greater at earlier times up to $24 \mathrm{~h}$, but the effect was gradually diminished thereafter. The HIFU effect on enhancing tumor uptake was substantiated by nuclear imaging studies. HIFU also increased the uptake of the antibody in surrounding tissues, but the net increase was marginal compared with the increase in tumor uptake. This study demonstrates that pulsed-HIFU significantly enhances the delivery of (111)In-MX-B3 in human epidermoid tumors xenografted in nude mice. The results of this pilot study warranted further evaluation of other treatment regimens, such as repeated HIFU exposures for greater delivery enhancement of antibodies labeled with cytotoxic radioisotopes or pulsed-HIFU exposure in addition to a combined therapy of (90)Y-B3 and taxol to enhance the synergistic effect.

\section{CONCLUSION}

In summary, even though today only nine drugs enhance are administered transdermally in general practice, with the advent and development of ultrasound mediated transdermal transport, patches may soon become the name of the game. Besides, taking into account the varied possible applications of sonophoretic TDT in fields of biotechnology and genetic engineering, we can envision a whole gamut of newer technologies and products in the foreseeable future.

Current evidence [9] suggests that sonophoresis are promising methods of enhancing topical delivery of both dermatologic and nondermatologic drugs (Table II). These methods may enable precise control of transdermal drug delivery rates by varying ultrasound frequency. Further controlled studies of this modality are necessary to determine optimal technique and conditions for safe and efficacious utilization. Future interest may also focus on systemic phonophoretic delivery of peptide and protein drugs.

Studies on the physics of ULTS as an enhancer of transcutaneous drug delivery must also be continued. This research should be closely paralleled with research on the variables of ULTS delivery, the length of a treatment session, and the duration of phonophoresis treatments in terms of effectiveness of diffusion at the local as well as systemic level. This same research should be come out with asymptomatic subjects as well as those with pathology.

\section{ACKNOWLEDGMENTS}

José Juan Escobar-Chávez wishes to acknowledge the PROFIP/UNAM grant.

\section{REFERENCES}

[1]. Wood, R.W., Loomis, A.L. The physical and biological effects of high frequency sound waves of great intensity. Phil. Mag. 4: 417-436, 1927

[2]. Benwell AD, Bly S.H.P. Sources and applications of ultrasound. In: Repacholi MH, Grandolfo M, Rindi A, editors. Ultrasound: Medical application, biological effects and hazard potentials. New York: Plenum Press. pp. 29-47, 1987.

[3]. Skauen DM, Zentner GM. Phonophoresis. Int J Pharm 20:235-245,1984.

[4]. McElnay JC, Matthews MP, Harland R, McCafferty DF. 1985. The effect of ultrasound on the percutaneous absorption of lignocaine. $\mathrm{Br}$ J Clin Pharmacol 20:421-424, 1985.

[5]. Mitragotri S, Blanckschtein D, Langer R. Transdermal drug delivery using low frequency sonophoresis. Pharm Res 13:411-420, 1996.

[6]. Kost J, Mitragotri S, Gabbay RA, Pishko M, Langer R. Transdermal monitoring of glucose 
and other analytes using ultrasound. Nature Med 6:327-350, 2000.

[7]. Amit Joshi, Jaideep Raje. Sonicated transdermal drug transport. J. Control. Rel., 83:13-22, 2002.

[8]. Kushner IV, J., Blankschtein D., Langer R. Heterogeneity in skin treated with lowfrequency ultrasound. J. Pharm. Sci., 97:41194128, 2008.

[9]. ter Haar G. Therapeutic applications of ultrasound. Progress in Biophysics and Molecular Biology.93:111-129, 2007.

[10]. Lavon I., Kost J. Ultrasound and transdermal drug delivery. D.D.T., 9(15): 670-676, 2004.

[11]. Machet L., Boucaud B. Phonophoresis: efficiency, mechanisms and skin tolerance. Int. J. Pharm., 243:1-15, 2002.

[12]. Merino G., Kalia Y.N., Guy R.H. UltrasoundEnhanced Transdermal Transport. J.Pharm. Sci., 92:1125-1137, 2003.

[13]. Forslind B.A. Domain mosaic model of skin barrier. Acta Derm Venereol. 74:1-6, (1994)

[14]. Potts R.O., Francoeur M.L. The influence of stratum corneum morphology on water permeability. J Invest Dermatol. 96, 495-499, 1991.

[15]. Potts RO, Guy RH. Predicting skin permeability. Pharm. Res, 9(5): 663-669, 1992.

[16]. Ellias P.M. Epidermal barrier function: intercellular lamellar lipid structures, origin, composition and metabolism, J Control Rel. 15, 199-208, 1991.

[17]. Barry B.W. Dermatological Formulations: Percutaneous Absorption. In: Swarbick J, ed. Drugs and the Pharmaceutical Sciences. New York and Basel: Marcel Dekker, Inc. p.202, 1983.

[18]. Hadgraft J. Skin, the final frontier. Int J Pharm. 224 (1-2):1-18, 2001.

[19]. Guy R.H and Hadgraft J. Transdermal drug delivery. New York: Marcel Dekker, Inc., p. 123, 2003.

[20]. Walters K.A and Roberts M.S. Dermatological and transdermal formulations. New York: Marcel Dekker, Inc., p. 1-39, 2002.

[21]. Nevill A.M. The need to scale for differences in body size and mass: and explanation of Klieber's 0.75 mass exponent. Am Physiol Soc. 2870-2873, 1994.

[22]. Olvera-Martínez B.I, Cazares-Delgadillo J., Calderilla-Fajardo S.B., Villalobos-García R., Ganem-Quintanar A., Quintanar-Guerrero D. Preparation of polymeric nanocapsules containing octyl methoxycinnamate by the emulsification-diffusion technique: Penetration across the stratum corneum. J Pharm Sci. 94:1552-1559, 2005.
[23]. Escobar-Chávez J.J., Quintanar-Guerrero D., and Ganem-Quintanar A. In vivo skin permeation of sodium naproxen formulated in PF-127 gels: Effect of Azone ${ }^{\circledR}$ and Transcutol ${ }^{\circledR}$, Drug Develop Ind Pharm. 31:447-454, 2005.

[24]. Escobar-Chávez J.J., López-Cervantes M., Naïk A., Kalia Y.N., Quintanar-Guerrero D., GanemQuintanar A. Applications of the thermoreversible Pluronic F-127 gels in pharmaceutical formulations, $J$ Pharm Pharmaceut Sci. 9(3):339-358, 2006.

[25]. Miyazaki S, Yokouchi Ch, Nakamura T, Hashiguchi N, Hou W-M, and Takada M. Pluronic F-127 gels as a novel vehicle for rectal administration of indomethacin, Chem Pharm Bull.34, 1801-1808, 1986.

[26]. Chi SCh, Do K, Tan HK, and Chun HW. Antiinflammatory and analgesic transdermal gel, United States Patents., Patent number 5,527,832, 1996.

[27]. Fang JY, Leu YL, Wang YY, and Tsai YH. In vitro topical application and in vivo pharmacodynamic evaluation of nonivamide hydrogels using Wistar rat as an animal model, Eur. J Pharm Sci. 15(5):417-423, 2002.

[28]. Shin SC, Cho CW, and Oh IJ. Effects of non ionic surfactants as permeation enhancers towards piroxicam from the poloxamer gel through rat skins, Int J Pharm. 222(2), 199-203, 2001.

[29]. Liaw J, and Lin Y-Ch. Evaluation of poly(ethylene oxide)-poly(propylene oxide)poly(ethylene oxide) (PEO-PPO-PEO) gels as a release vehicle for percutaneous fentanyl, $J$ Control. Rel. 68:273-282, 2000.

[30]. Wang YY, Hong CT, Chiu WT, and Fang JY. In vitro and in vivo evaluations of topically applied capsaicin and nonivamide from hydrogels, Int $J$ Pharm. 224,1-2, 2001.

[31]. Kattan El, A. F., Asbill, C. S., Kim, N., and Michniak, B. B. Effect of formulation variables on the percutaneous permeation of ketoprofen from gel formulations, Drug Deliv. 7,3, 2000.

[32]. Curdy C, Kalia YN, Naïk A, Guy RH. Piroxicam delivery into human stratum corneum in vivo: iontophoresis versus passive diffusion. J Control Rel. 76, 73-79, 2001.

[33]. Mattorano DA, Kupper LL, Nylander-French LA. Estimating dermal exposure to jet fuel (naphthalene) using adhesive tape strip samples. Ann Occup Hyg. 48(2): 139-146, 2004.

[34]. Chao Y-Ch, Nylander-French LA. Determination of Keratin Protein in a Tapestripped Skin Sample from Jet Fuel Exposed Skin. Ann Occup Hyg. 48(1):65-73, 2004. 
[35]. Zagzebski JA. Physics of diagnostic ultrasound. In: Lading DE, Potts L, editors. Essentials of ultrasound physics. St. Louis, MO: Mosby-Year Book, pp. 1-19, 1996.

[36]. Weyman AE. Physical principles of ultrasound. In: Weyman AE, editor. Principles and practice of echocardiography. Philadelphia: Lea and Febiger, pp.3-28, 1994.

[37]. Clarke, L., Edwards, A., Graham, E. Acoustic streaming: an in vitro study. Ultrasound Med. Biol. 30, 559-562, 2004.

[38]. K. Tachibana, S. Tachibana, Application of ultrasound energy as a new drug delivery system, Jpn. J. Appl. Phys. 38(1):3014-3019, 1999.

[39]. S. Mitragotri, D.A. Edwards, D. Blankschtein, R. Langer, A. Mechanistic study of ultrasonically-enhanced transdermal drug delivery, J. Pharm. Sci. 84(6):697-706, 1995.

[40]. T.J. Mason, J.P. Lorrimer, SonochemistryTheory, applications and uses of ultrasound in chemistry, Ellis Horwood, 1988.

[41]. T.J. Mason, Practical Sonochemistry: User's Guide To Professor Application in Chemistry and Chemical Engineering, Ellis Horwood, 1991.

[42]. Mitragotri, S., Kost J. Low-frequency sonophoresis: a noninvasive method of drug delivery and diagnostics, Biotechnol. Prog. 16:488-492, 2000.

[43]. Tezel A., Sens A., Mitragotri S. Investigation of the role of cavitation in low-frequency sonophoresis using acoustic spectroscopy, J. Pharm. Sci. 91(2):444-453, 1998.

[44]. Tang H., Mitragotri S., Blankschtein D., Langer R. Theoretical description of transdermal transport of hydrophilic permeants: application to low frequency sonophoresis, J. Pharm. Sci., 90(5):543-566, 2001.

[45]. Lubbers, J., Hekkenberg, R.T, Bezemer, R.A.Time to threshold (TT), a safety parameter for heating by diagnostic ultrasound. Ultrasound Med. Biol. 29, 755-764, 2003.

[46]. Wells, P.N.T. Physics of ultrasound. In Ultrasonic Exposimetry (Ziskin, M. and Lewin, P., eds), p. 35, CRC Press., 1993.

[47]. Lavon I., Grossman N., Kost J., Kimmel E., Enden G. Bubble growth within the skin by rectified diffusion might play a significant role in sonophoresis. J. Control. Rel., 117:246-255, 2007.

[48]. Lavon I., Grossman N., Kost J. The nature of ultrasound-SLS synergism during enhanced transdermal transport. J. Control. Rel., 107:484494, 2005.
[49]. Nanda A, Nanda S, Ghilzai N.M. Current developments using emerging transdermal technologies in physical enhancement methods. Curr. Drug Deliv., 3(3):233-242, 2006.

[50]. Daniel G., Kassan, M.D., Agnes M., Lynch, B.S., Matthew J., Stiller, M.D. Physical enhancement of dermatologic drug: Iontophoresis and phonophoresis delivery: J. Am. Academ. Dermatol.,34(4):657-666, (1996).

[51]. Maloney M, Bezzant JL, Stephen RL. Iontophoreric administration of lidocaine anesthesia in office practice. J Dermatol Surg Oncol., 18:937-940, 1992.

[52]. Meshali M.M., Abdel-Aleem H.M., Sakr F.M., Nazzal S., El-Malah Y. In vitro phonophoresis: effect of ultrasound intensity and mode at high frequency on NSAIDs transport across cellulose and rabbit skin membranes, Pharmazie, 63(1):49-53, 2008.

[53]. Miyazaki S, Mizuoka H, Kohata Y, Takada M. External control of drug release and penetration. Enhancing effect of ultrasound on the transdermal absorption of indomethacin from an oinment in rats, Chem. Pharm. Bull (Tokyo), 40(10):2826-2830, 1992.

[54]. Tiwari S.B., Pai R.M., Udupa N. Influence of ultrasound on the percutaneous absorption of ketorolac tromethamine in vitro across rat skin, Drug Deliv., 11(1):47-51, 2004.

[55]. Yang JH, Kim TY, Lee JH, Yoon SW, Yang $\mathrm{KH}$, Shin SC. Anti-hyperalgesic and antiinflammatory effects of ketorolac tromethamine gel using pulsed ultrasound in inflamed rats. Arch. Pharm.Res., 31(4):511-517, 2008.

[56]. Serikov NP. Efficacy of ibuprofen (nurofen gel) ultraphonophoresis for pain in ostheoarthritis. Ter. Arkh., 79(5):79-81, 2007.

[57]. Cabak A, Maczewska M, Lyp M, Dobosz J, Gasiorowska U. The effectiveness of phonophoresis with ketoprofen in the treatment of epocondylopathy. Ortop. Traumatol. Rehabil., 37(6):660-665, 2005.

[58]. Rosim GC, Barbieri CH, Lanças FM, Mazzer N. diclofenac phonphoresis in human volunteers. Ultrasound Med. Biol., 31(3):337-343, 2005.

[59]. Hippius M., Uhlemann C., Smolenski U., Schreiber U., Reissig S., Hoffmann A. In vitro investigations of drug release and penetration enhancing effect of ultrasound on transmembrane transport of flufenamic acid, Int. Clin. Pharmacol. Ther., 36(2):107-111, 1998.

[60]. Tachibana K, Tachibana S. Use of ultrasound to enhance the local anesthetic effect of topically applied aqueous lidocaine, Anesthesiology, 78(6):1091-1096, 1993. 
[61]. Hehn B, Moll F. Phonophoretic permeation of procaine hydrochloride through and MDCK cell monolayer. Pharmazie, 51:341-345, 1996.

[62]. Kim TY, Jung DI, Kim YI, Yang JH, Shin SC. Anesthetic effects of lidocaine hydrochloride gel using low frequency ultrasound of $0.5 \mathrm{MHz}$. J. Pharm. Pharm. Sci., 10(1):1-8, 2007.

[63]. Liu H., Li S., Pan W., Wang Y., Han F., Yao H Investigation into the potential of low-frequency ultrasound facilitated topical delivery of Cyclosporin A. Int. J. Pharm., 326:32-38, 2006.

[64]. Pietro SantoiannI, Massimiliano Nino, and Gabriella Calabro. Intradermal drug delivery by low frequency sonophoresis $(25 \mathrm{KHz})$. Dermatology on line Journal., 10(2):24-29.

[65]. Ragelis Siu. Tetracycline penetration into tissue by modified electro and phonophoretic methods, Antibiotiki, 26(9): 699-703, 1981.

[66]. Aoi A, Watanabe Y, Mori S, Takahashi M, Vassaux G, Kodama T.herpes simplex virus thymidine kinase mediated suicide gene therapy using nano/microbubbles and ultrasound. Ultrasound Med. Biol., 34(39):425-434, 2007.

[67]. Meidan VM, Walmsley AD, Docker MF, Irwin WJ. Ultrasound enhanced diffusion into coupling gel during phonophoresis of 5fluorouracil. 185(2): 205-213, 1999.

[68]. Luis J, Park EJ, Meyer RJ, Smith NB. Rectangular cymbal arrays for improved ultrasonic transdermal insulin delivery. J. Acoust. Soc. Am., 122(4):2022-2030, 2007.

[69]. Lee S, Snyder B, Newnham RE, Smith NB. Noninvasive ultrasonic transdermal insulin delivery in rabbits using the light weight cymbal array. Diabetes Techno. Ther., 6(6): 808-815, 2004.

[70]. Smith N.B., Lee S., Shung K.K. Ultrasoundmediated transdermal in vivo transport of insulin with low profile cymbal arrays. Ultrasound med. Boil., 29(8):1205-1210, 2003.

[71]. Saliba S., Mistry D.J., Perrin D.H., Gieck J., Weltman A. Phonophoresis and the absorption of dexamethasone in the presence of an occlusive dressing. Journal of Athletic Training, 42(3):349-354, 2007.

[72]. Byl N.N., McKenzie A., Halliday B., Wong T., O'Connell $\mathrm{J}$ The effects of phonophoresis with corticosteroids a controlled pilot study, J. Orthop. Sports Phys.Ther., 18(5):590-600, 1993.

[73]. Akinbo S.R., Aiyejusunle C.B., Akinyemi O.A., Adesegun S.A., Danesi M.A. Comparison of the therapeutic efficacy of phonophoresis and iontophoresis using dexamethasone sodium phosphate in the management of patients with knee ostheoarthritis. Niger. Postgrad. Med, J., 14(3):190-194, 2007.

[74]. Yang J.H., Kim D.K., Yun M.Y., Kim T.Y., Shin S.C. Transdermal delivery system of triamcinolone acetonide from a gel using phonophoresis. Arch. Pharm. Res., 29(5):412427, 2006.

[75]. Meidan V.M., Docker M.F., Walmsley A.D., Irwin W.J. Phonophoresis of hydrocortisone with enhancers: an acoustically defined model. Int. J. Pharm., 170:157-168,1998.

[76]. Machet L., Pinton J., Patat F., Arbeille B., Pourcelot L., Vaillant L. In vitro phonophoresis of digoxin across hairless mice and human skin: thermal effect of ultrasoundInternational Journal of Pharmaceutics 133 39-45, 1996.

[77]. McElnay J.C., Benson H.A., Harland R., Hadgraft J. Phonophoresis of methyl nicotinate. A preliminary study to elucidate the mechanism of action, Pharm. Res., 10(12):1726-1731, 1993.

[78]. El-Kamel A.H., Al-Fagih I.M., Alsarra I.A. Effect of sonophoresis and chemical enhancers on testosterone transdermal delivery from solid lipid microparticles:an in vitro study, Curr. Drug Deliv., 5(1):20-26, 2008.

[79]. Park S.R., Jang K.W.,Park S-H.,Cho H-S., Jin Ch-Z., Choi M.J., Chung S.I., Min B.H. The effect of sonication on simulated osteoarthritis part I: effect of $1 \mathrm{MHz}$ ultrasound on uptake of hyaluronan into the rabbit synovium. Ultrasound Med. Biol., 31(11):1551-1558, 2005

[80]. Morimoto Y., Mutoh T.M., Ueda H., Fang L., Hirayama K., Atobe M., Kobayashi D. Elucidation of the transport pathway in hairless rat skin enhanced by low-frequency sonophoresis based on the solute-water transport relationship and confocal microscopy. J.Control Rel., 103:587-597, 2005.

[81]. Tezel H., Dokka S., Kelly S., Hardee G. E., Mitragotri.S. Topical delivery of anti-sense oligonucleotides using low-frequency sonophoresis. Pharm. Res., 21(12):2219-2225, 2004.

[82]. Boucaud A., Machet L., Arbeille B., Machet M.C., Sournac M., Mavon, A., Patat F., Vaillant L. In vitro study of low-frequency ultrasoundenhanced transdermal transport of fentanyl and caffeine across human and hairless rat skin. Int. J. Pharm., 228, 69-77, 2001.

[83]. Menon G.K., Price L. F., Bommannan B., Elias P.M., Feingold K. R. Selective obliteration of the epidermal calcium gradient leads to enhanced lamellar body secretion.J. Invest. Dermatol., 102(5):789-795,1994.

[84]. Ng G.Y., Wong R.Y. Ultrasound phonophoresis of panax notoginseng improves the strength of 
repairing ligament: a rat model. Ultrasound Med. Biol., 34(12):1919-1923, 2008.

[85]. Kushner J. IV., Blankschtein D., Langer R. Experimental demonstration of the existence of highly permeable localized transport regions in low-frequency sonophoresis. J. Pharm. Sci., 93:2733-2745, 2004.

[86]. Alvarez-Román R., Merino G., Kalia Y.N., Naik A., Guy R.H. Skin permeability enhancement by low frequency sonophoresis: Lipid extraction and transport pathways. J. Pharm. Sci., 92, 1138-1146, 2003.

[87]. Cancel L.M., Tarbell J.M., Ben-Jebria A. Fluorescein permeability and electrical resistance of human skin during low frequency ultrasound application. J Pharm Pharmacol., 56:1109-1118, 2004.
[88]. Paliwal S., Menon G.K., Mitragotri S. Lowfrequency sonophoresis: ultrastructural basis for stratum corneum permeability assessed using quantum dots. J. Invest. Dermatol., 126, 10951101, 2006.

[89]. Larkin JO, Casey GD, Tangney M, Cashman J, Collins CG, Soden DM, O'Sullivan GC. Effective tumor treatment using optimized ultrasound mediated delivery of bleomycin. Ultrasound Med. Biol., 34(3): 406-413, 2008.

[90]. Khaibullina A, Jang BS, Sun H, Le N, Yu S, Frenkel V, Carrasquillo JA, Pastan I, Li K.C, Paik CH.Pulsed high intensity focused ultrasound enhances uptake of radiolabeled monoclonal antibody to human epidermoid tumor in nude mice. J. Nucl. Med., 49(2):295302, 2008. 